\title{
Multiple protostellar systems
}

\section{A deep near infrared survey of Taurus and Ophiuchus protostellar objects ${ }^{\star}$}

\author{
G. Duchêne $e^{1,2}$, J. Bouvier ${ }^{2}$, S. Bontemps ${ }^{3}$, P. André ${ }^{4}$, and F. Motte ${ }^{4}$ \\ 1 Department of Physics \& Astronomy, UCLA, Los Angeles, CA 90095-1562, USA \\ 2 Laboratoire d'Astrophysique de Grenoble, Université Joseph Fourier, BP 53, 38041 Grenoble Cedex 9, France \\ e-mail: gaspard. duchene@obs.ujf-grenoble.fr \\ 3 Observatoire de Bordeaux, BP 89, 33270 Floirac, France \\ ${ }^{4}$ CEA/DSM/DAPNIA, Service d'Astrophysique, CEA Saclay, 91191 Gif-sur-Yvette Cedex, France
}

Received 1 May 2004 / Accepted 21 July 2004

\begin{abstract}
We performed a deep infrared imaging survey of 63 embedded young stellar objects (YSOs) located in the Taurus and Ophiuchus clouds to search for companions. The sample includes Class I and flat infrared spectrum protostellar objects. We find 17 companions physically bound to 15 YSOs with angular separations in the range $0.8-10^{\prime \prime}(110-1400 \mathrm{AU})$ and derive a companion star fraction of $23 \pm 9 \%$ and $29 \pm 7 \%$ for embedded YSOs in Taurus and Ophiuchus, respectively, about twice as large as that found among $\mathrm{G}$ dwarfs in the solar neighborhood. Therefore, binary and multiple protostellar systems are a very frequent outcome of the fragmentation of prestellar cores. In spite of different properties of the clouds and especially of the prestellar cores, the fraction of wide companions, $27 \pm 6 \%$ for the combined sample, is identical in the two star-forming regions. This suggests that the frequency and properties of wide multiple protostellar systems are not very sensitive to specific initial conditions. Comparing the companion star fraction of the youngest YSOs still surrounded by extended envelopes to that of more evolved YSOs, we find evidence for a possible evolution of the fraction of wide multiple systems, which seems to decrease by a factor of about two on a timescale of $\sim 10^{5} \mathrm{yr}$. For the first time it is possible to confront the result of a multiplicity survey of a nearly complete population of embedded YSOs at an age of $\sim 10^{5} \mathrm{yr}$ to numerical simulations of molecular cloud collapse which, after a few free fall times, reach this evolutionary stage. Somewhat contrary to model predictions, we do not find evidence for a sub-clustering of embedded sources at this stage on a scale of a few $100 \mathrm{AU}$ that could be related to the formation of small- $N$ protostellar clusters. Possible interpretations of this discrepancy are discussed.
\end{abstract}

Key words. stars: formation - stars: binaries: visual - stars: pre-main sequence

\section{Introduction}

Giant molecular clouds produce stars in a multi-step process that needs to be understood in order to account for the general properties of stars, such as the stellar mass function or the properties of multiple systems in the Galaxy. First, the molecular cloud fragments into individual cores that subsequently collapse to form individual stars. In many cases, however, those cores fragment a second time during protostellar collapse (in the following, we refer to this phenomenon as "core dynamical fragmentation"), which leads to the formation of binary and higher order multiple systems. The extent to which this process depends upon initial physical conditions of the cloud is currently under debate. Numerical simulations suggest that the dynamical fragmentation process is strongly dependent on the initial conditions, such as cloud rotation, gas temperature or magnetic field strength (e.g., Bonnell et al. 1992; Durisen \& Sterzik 1994; Boss 2002). With several star-forming

* Based on observations obtained at the Canada-France-Hawaii Telescope and at the European Southern Observatory, La Silla, Chile. regions located within $500 \mathrm{pc}$ of the Sun that range from relatively quiescent zones such as the Taurus-Auriga cloud to the dense cluster-forming Orion Nebula, a number of observational tests can be performed to search for environment-induced effects.

The study of the collapse and core fragmentation processes usually focuses on two main approaches. Investigation of prestellar cores within molecular clouds provides insight in the initial conditions and the very early phases of the collapse while the analysis of populations of pre-main sequence (T Tauri) objects reveals their outcome. Due to the limited spatial resolution of the single-dish millimeter images from which prestellar core properties are derived, it is usually not possible to probe spatial scales of a few tens or hundreds AU, typical of most multiple systems. On the other hand, T Tauri stars have not necessarily preserved their original properties after the dissipation of their collapsing envelope. Studying more deeply embedded young stellar objects (YSOs), which are assumed to be much younger than T Tauri stars, at high spatial resolution 
$\left(\leq 1^{\prime \prime}\right)$ offers an opportunity to sample a crucial intermediate stage between prestellar cores and pre-main sequence stars.

Recent studies have revealed ranges in the properties of prestellar cores as well as of the young populations resulting from the star formation process, with significant differences between star-forming regions. We summarize here some of the most notable ones, with emphasis on the Taurus and Ophiuchus molecular clouds which constitute the focus of the present study. First of all, the Taurus molecular cloud forms only a few tens of objects in $\sim 1$ pc-radius groups (Gomez et al. 1993) and is frequently referred to as the prototype of "isolated" star formation. It is believed that most stars form in much richer, cluster-like structures with several hundred objects within a 1 pc-radius (e.g., Carpenter 2000; Adams \& Myers 2001). While the Orion Trapezium cluster is a well known example of this "clustered" mode of star formation, the Ophiuchus molecular cloud also belongs to this category, as it hosts several hundred objects with an average spatial density of order $10^{3}$ stars $/ \mathrm{pc}^{3}$ (Bontemps et al. 2001; Allen et al. 2002). Taurus and Ophiuchus are therefore excellent candidates for investigating whether the star formation process is sensitive to environmental conditions.

Millimeter continuum mapping of both star-forming regions has revealed clear differences in the properties of their prestellar cores. While cores in Taurus appear to have a radius as large as 10000-20000 AU and smoothly merge into the ambient molecular cloud (Motte \& André 2001), those found in Ophiuchus exhibit a sharp boundary at a radius of order 5000 AU (Motte et al. 1998), possibly due to external perturbations in this denser environment. The derived envelope masses, bolometric luminosities and density profiles of prestellar cores also differ between the two molecular clouds. Likewise, at a slightly later stage, the circumstellar envelopes of embedded protostars in Ophiuchus are smaller than those of their Taurus counterparts (Motte et al. 1998). This led Motte \& André (2001) to state that "the beginning of protostellar evolution is suggested to be more violent (...) in protoclusters compared to regions of distributed star formation like Taurus". In other words, the initial conditions for core fragmentation appear to be significantly different in the Taurus and Ophiuchus molecular clouds.

Multiple systems are useful tracers of the core fragmentation process. Systematic surveys have revealed that young stellar populations in dense stellar clusters (Orion Trapezium, IC 348, Pleiades) exhibit multiplicity rates similar to field stars whereas populations of T Tauri stars located in more distributed associations can possess up to twice as many companions (e.g., Duchêne 1999). However, it must be noted that the frequency of visual companions to T Tauri stars in the Taurus and Ophiuchus clouds are identical, both exhibiting a strong excess of multiple systems, i.e., that the latter behaves in the same way as "loose associations" in this respect although it is an example of clustered star formation.

The lower multiplicity rate in dense clusters does not necessarily imply that the core fragmentation process itself is environment-dependent. For instance, Kroupa et al. (1999) have performed $\mathrm{N}$-body simulations to follow the dynamical evolution of young stellar clusters and showed that the observed properties of multiple systems in these populations are consistent with an initial set of multiple systems identical to loose T Tauri associations. In clusters, direct dynamical encounters between systems are able to disrupt a fraction of the initial (wide) systems over timescales as short as 1 Myr (e.g., Kroupa 1995a), thus rapidly bringing the multiplicity rate down to the observed value.

In order to probe the outcome of the fragmentation process before it is dynamically modified, at least in clustered environments, it is therefore necessary to study younger YSOs than T Tauri stars. In this study, we present a systematic search for close companions among Class I sources and slightly more evolved "flat-spectrum" objects in Taurus-Auriga and Ophiuchus. These objects exhibit a flat or rising spectral energy distribution from the near to the mid infrared and are believed to still be heavily embedded within a collapsing envelope. On a statistical basis, their estimated age is of order $10^{5} \mathrm{yrs}$ (e.g. Kenyon \& Hartmann 1995). Because they are seen through a high column density of dust, these objects are almost undetected in the visible and deep near infrared imaging is required to look for close companions. Recently, Haisch et al. (2004) reported the results of an imaging survey of samples of embedded YSOs located in several nearby molecular clouds and derived a multiplicity rate over a separation range 300-2000 AU similar to that of more evolved T Tauri stars but they did not reach a conclusion regarding possible differences between various starforming regions. Our survey differs from theirs in two respects. Firstly, we focus on homogeneous and nearly complete samples of embedded YSOs in two star-forming regions only, Taurus and Ophiuchus, in order to be able to contrast at a significant statistical level the multiplicity rate resulting from the isolated and clustered star formation modes. Second, the 0.6" angular resolution of the present survey allows us to explore closer systems down to a separation of $110 \mathrm{AU}$ at the distance of these two clouds (140 pc, Bertout et al. 1999; Bontemps et al. 2001).

This paper is organized as follows: in Sect. 2, we present our samples and deep infrared observations obtained at CFHT and ESO/NTT. Candidate visual companions detected in the images are presented in Sect. 3 and physical companions to embedded YSOs are identified on statistical grounds, thus allowing us to establish the multiplicity frequency of embedded YSOs in the Taurus and Ophiuchus clouds. In Sect. 4, we discuss the results and their implications regarding the fragmentation of prestellar cores in the two star-forming regions, and compare the multiplicity frequency we derive for embedded Class I and flat spectrum sources to that of both younger Class 0 sources and older Class II-III T Tauri stars in the same clouds in order to investigate the dynamical evolution of protostellar systems on a timescale of a few Myr. Finally, we summarize our results in Sect. 5.

\section{Sample selection and observations}

We have built our samples from the object lists from Motte \& André (2001) in Taurus and Bontemps et al. (2001) in Ophiuchus. The list of our targets is presented in Tables 1 and 2 , together with their near-infrared photometry and nearto mid-infrared spectral index. The latter is used to distinguish 
Table 1. Sample of protostars surveyed in Taurus. The infrared photometry and the spectral indices are taken from Kenyon \& Hartmann (1995), except for IRAS $04191+1523$, whose $J H K$ photometry is from the 2MASS database and classification from Motte \& André (2001). The bolometric luminosities are from Motte \& André (2001). IRAS did not resolve the systems IRAS 04108+2803 AB and IRAS 04181+2654 AB and we assigned the same spectral index, based on unresolved photometry, to both components and split evenly the bolometric luminosity of the later system. The classification of each source is based on the infrared spectral indices (see text); "I" stands for Class I sources and "FS" for flat spectrum sources. The last three columns indicate whether the source i) has at least one visual companion within 10", ii) is surrounded by an extended nebulosity in our images (see also Park \& Kenyon 2002), and iii) has an extended (>1000 AU) envelope as seen in millimeter continuum mapping (from Motte \& André 2001).

\begin{tabular}{lcccccccccc}
\hline Object & $J$ & $H$ & $K$ & $\alpha_{\text {IR }}^{2-12}$ & $\alpha_{\text {IR }}^{2-25}$ & Class & $L_{\text {bol }}\left(L_{\odot}\right)$ & Comp? & Opt neb? & Mm env? \\
\hline IRAS 04016+2610 & 13.62 & 11.67 & 9.33 & 1.06 & 1.02 & $\mathrm{I}$ & 3.7 & $\mathrm{~N}$ & $\mathrm{Y}$ & $\mathrm{N}$ \\
IRAS 04108+2803 B & 16.25 & 13.25 & 11.12 & 0.50 & 0.62 & $\mathrm{FS}$ & 0.6 & $\mathrm{~N}$ & $\mathrm{~N}$ & $\mathrm{~N}$ \\
IRAS 04113+2758 & 11.29 & 9.15 & 7.79 & -0.01 & 0.12 & $\mathrm{FS}$ & $>1.6$ & $\mathrm{Y}$ & $\mathrm{N}$ & $\mathrm{Y}$ \\
IRAS 04158+2805 & 13.15 & 11.92 & 10.80 & 1.47 & 0.72 & $\mathrm{I}$ & $>0.4$ & $\mathrm{~N}$ & $\mathrm{Y}$ & $\mathrm{N}$ \\
IRAS 04169+2702 & 16.87 & 13.83 & 11.22 & 0.89 & 1.10 & $\mathrm{I}$ & 0.8 & $\mathrm{~N}$ & $\mathrm{Y}$ & $\mathrm{Y}$ \\
IRAS 04181+2655 & 15.46 & 12.74 & 10.54 & 0.60 & 0.55 & $\mathrm{I}$ & 0.4 & $\mathrm{~N}$ & $\mathrm{~N}$ & $\mathrm{~N}$ \\
IRAS 04181+2654 A & 15.90 & 12.74 & 10.58 & -0.00 & 0.10 & $\mathrm{FS}$ & 0.25 & $\mathrm{~N}$ & $\mathrm{~N}$ & $\mathrm{Y}$ \\
IRAS 04181+2654 B & 18.11 & 13.64 & 10.81 & -0.00 & 0.10 & $\mathrm{FS}$ & 0.25 & $\mathrm{~N}$ & $\mathrm{~N}$ & $\mathrm{Y}$ \\
IRAS 04191+1523 & 16.74 & 14.43 & 12.26 & & & $\mathrm{I}$ & 0.5 & $\mathrm{Y}$ & $\mathrm{Y}$ & $\mathrm{Y}$ \\
IRAS 04239+2436 & 15.61 & 12.96 & 10.58 & 1.27 & 1.15 & $\mathrm{I}$ & 1.3 & $\mathrm{~N}$ & $\mathrm{~N}$ & $\mathrm{Y}$ \\
IRAS 04248+2612 & 12.76 & 11.39 & 10.65 & -0.09 & 0.48 & $\mathrm{FS}$ & 0.4 & $\mathrm{Y}$ & $\mathrm{Y}$ & $\mathrm{Y}$ \\
IRAS 04260+2642 & 14.64 & 12.96 & 11.63 & 0.16 & 0.25 & $\mathrm{FS}$ & 0.1 & $\mathrm{~N}$ & $\mathrm{~N}$ & $\mathrm{~N}$ \\
IRAS 04263+2426 & 10.84 & 9.06 & 7.80 & 1.02 & 0.74 & $\mathrm{I}$ & 7.0 & $\mathrm{Y}$ & $\mathrm{Y}$ & $\mathrm{Y}$ \\
IRAS 04264+2433 & 13.63 & 12.07 & 11.09 & 0.67 & 0.98 & $\mathrm{I}$ & 0.5 & $\mathrm{~N}$ & $\mathrm{~N}$ & $\mathrm{~N}$ \\
IRAS 04295+2251 & 13.62 & 11.11 & 9.55 & 0.11 & 0.21 & $\mathrm{FS}$ & 0.6 & $\mathrm{~N}$ & $\mathrm{~N}$ & $\mathrm{~N}$ \\
IRAS 04302+2247 & 14.20 & 12.35 & 11.07 & & 0.15 & $\mathrm{FS}$ & 0.3 & $\mathrm{~N}$ & $\mathrm{Y}$ & $\mathrm{N}$ \\
IRAS 04325+2402 & & 13.02 & 11.03 & & 0.79 & $\mathrm{I}$ & 0.9 & $\mathrm{Y}$ & $\mathrm{Y}$ & $\mathrm{Y}$ \\
IRAS 04361+2547 & 16.07 & 12.88 & 10.55 & 1.27 & 1.55 & $\mathrm{I}$ & 3.7 & $\mathrm{~N}$ & $\mathrm{Y}$ & $\mathrm{Y}$ \\
IRAS 04365+2535 & 17.28 & 13.39 & 10.62 & 1.08 & 1.27 & $\mathrm{I}$ & 2.4 & $\mathrm{~N}$ & $\mathrm{Y}$ & $\mathrm{Y}$ \\
IRAS 04381+2540 & 16.21 & 14.32 & 12.00 & 1.20 & 1.31 & $\mathrm{I}$ & 0.7 & $\mathrm{~N}$ & $\mathrm{Y}$ & $\mathrm{N}$ \\
IRAS 04385+2550 & 12.61 & 10.72 & 9.65 & 0.14 & 0.18 & $\mathrm{FS}$ & $>0.4$ & $\mathrm{~N}$ & $\mathrm{~N}$ & $\mathrm{~N}$ \\
IRAS 04489+3042 & 13.74 & 11.58 & 10.11 & 0.18 & 0.31 & $\mathrm{FS}$ & 0.3 & $\mathrm{~N}$ & $\mathrm{~N}$ \\
\hline
\end{tabular}

between Class I, flat-spectrum and T Tauri (Class II) sources, where we adopted $\alpha_{\mathrm{IR}}=0.55$ and $\alpha_{\mathrm{IR}}=-0.05$ thresholds between these categories (see Bontemps et al. 2001). Note that because of the large line-of-sight extinctions towards some of our targets, the use of the observed infrared spectral index is not completely secure. While there might be a few embedded $\mathrm{T}$ Tauri stars in our sample, especially among the objects classified as flat spectrum, we consistently use the observed spectral index to estimate each object's nature since we cannot estimate the extinction to each individual source. Overall, we have observed all known sources in both areas with the exception of two Class I sources and one flat-spectrum object in Taurus, all of which are in the crowded vicinity of HL Tau, to the south of the molecular cloud. Our sample therefore includes 22 protostellar sources in Taurus and 41 in Ophiuchus, in the magnitude range $7.5 \leq K \leq 14.5$ with a median of $K \sim 11$ mag. A nearinfrared color-color diagram of all our targets is presented in Fig. 1, which shows that most sources have redder colors than $\mathrm{T}$ Tauri stars, even if extinction is taken into account.

We conducted most of our observations with the widefield infrared camera CFHT-IR (Starr et al. 2000) at the Canada-France-Hawaii Telescope, a $1024 \times 1024$ detector which offers a pixel scale of $0.211 /$ pixel and a total field-of-view of 3'6. The Ophiuchus images were obtained on
June, 11th and 12th 2001, while the Taurus-Auriga dataset was obtained on October, 29th and 30th 2001. Each target was systematically surveyed in the $K$ band, where these protostars are brighter. In some multiple systems we obtained follow-up $H$ and $J$ images to compare the near-infrared colors of the primary to its candidate companions. For each field a sequence of four images was obtained, each of them being cubes of four exposures. Individual integration times ranged from $0.1 \mathrm{~s}$ to $40 \mathrm{~s}$ at $K$ and up to $1 \mathrm{~min}$ at $H$ and $J$. In some Ophiuchus fields, we obtained two sets of images at the same location, with a short and a long integration time in order to observe with similar contrasts both bright and faint targets in crowded fields. The median value and standard deviation of the full width at half maximum $(F W H M)$ measured on a sample of single stars throughout the runs are $0{ }^{\prime} 60 \pm 00^{\prime} 07$ and 0 . $^{\prime} 65 \pm 0$. ' 10 for the Ophiuchus and Taurus datasets respectively.

On March, 10th 2000, we obtained additional images of the L 1689 area, a core located to the south of the main cores of the Ophiuchus molecular cloud, with ESO's New Technology Telescope at la Silla Observatory. We used SOFI, which has a $1024 \times 1024$ detector, a 0!'288/pixel scale and 4'9 field-of-view centered on the known T Tauri star L 1689-IRS 5. With all three $J, H$ and $K$ filters, we obtained a sequence of $10 \times 1.18 \mathrm{~s}$ exposures using a random jittering pattern in a $20^{\prime \prime} \times 20^{\prime \prime}$ box. 
Table 2. Sample of protostars surveyed in Ophiuchus. ISO numbers, infrared spectral indices and bolometric luminosities are from Bontemps et al. (2001), while the near-infrared magnitudes are from Barsony et al. (1997), Greene et al. (1994) and the 2MASS database (2MASS uses a $K$ s filter rather than a $K$ filter; the difference in magnitude for an object can reach a few tenths of a magnitude). The source marked with $\mathrm{a} \dagger$ symbol has an infrared spectral index typical of a normal Class II source but Ressler \& Barsony (2001) have shown that this system contains a Class I source and it is classified as such here. The last three columns have the same meaning as in Table 1. The existence of an envelope is taken either from André \& Montmerle (1994) and Motte et al. (1998) or from our reexamination of the mosaic presented in the latter. (Note that this classification is more uncertain than in Taurus due to the smaller size of protostellar envelopes in Ophiuchus. Particularly uncertain cases are flagged with a “?”.)

\begin{tabular}{|c|c|c|c|c|c|c|c|c|c|c|}
\hline ISO \# & Object & $J$ & $H$ & $K$ & 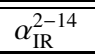 & Class & $L_{\mathrm{bol}}\left(L_{\odot}\right)$ & Comp? & Opt neb? & Mm env? \\
\hline 21 & CRBR 12 & $>17.00$ & 15.58 & 12.04 & 0.91 & I & 0.42 & $\mathrm{~N}$ & $\mathrm{~N}$ & $\mathrm{~N}$ \\
\hline 26 & CRBR 15 & 16.35 & 13.89 & 11.94 & 0.01 & FS & 0.083 & $\mathrm{~N}$ & $\mathrm{~N}$ & $\mathrm{~N}$ \\
\hline 29 & GSS 30, GY 6 & 13.89 & 10.83 & 8.32 & 1.20 & I & 21. & $\mathrm{~N}$ & $\mathrm{Y}$ & $\mathrm{Y} ?$ \\
\hline 31 & LFAM 1 & $>17.00$ & $>15.50$ & 13.59 & 1.08 & I & 0.13 & $\mathrm{Y}$ & $\mathrm{N}$ & $\mathrm{Y}$ \\
\hline 33 & GY 11 & 16.52 & 15.37 & 14.15 & 0.31 & FS & 0.011 & $\mathrm{Y}$ & $\mathrm{Y}$ & $\mathrm{N}$ \\
\hline 46 & VSSG 27, GY 51 & 16.69 & 13.46 & 10.72 & 0.17 & FS & 0.41 & $\mathrm{Y}$ & $\mathrm{N}$ & $\mathrm{N}$ \\
\hline 54 & GY 91, CRBR 42 & $>17.00$ & 16.40 & 12.51 & 0.70 & I & 0.17 & $\mathrm{~N}$ & $\mathrm{~N}$ & $\mathrm{Y}$ ? \\
\hline 65 & WL 12, GY 111 & 16.86 & 13.07 & 10.18 & 1.04 & I & 2.6 & $\mathrm{~N}$ & $\mathrm{Y}$ & Y? \\
\hline 70 & WL 2, GY 128 & $>17.00$ & 14.05 & 10.99 & 0.05 & FS & 1.4 & $\mathrm{Y}$ & $\mathrm{N}$ & $\mathrm{N}$ \\
\hline 75 & GY 144 & $>17.00$ & 15.75 & 13.46 & 0.20 & FS & 0.053 & $\mathrm{~N}$ & $\mathrm{~N}$ & $\mathrm{~N}$ \\
\hline 77 & GY 152 & $>17.00$ & $>15.75$ & 13.64 & 0.05 & FS & 0.052 & $\mathrm{~N}$ & $\mathrm{~N}$ & $\mathrm{~N} ?$ \\
\hline 85 & CRBR 51 & $>17.00$ & $>15.75$ & 14.00 & 0.03 & FS & 0.035 & $\mathrm{~N}$ & $\mathrm{~N}$ & $\mathrm{~N} ?$ \\
\hline 99 & LFAM 26, GY 197 & $>17.00$ & $>17.90$ & 14.63 & 1.25 & I & 0.064 & $\mathrm{~N}$ & $\mathrm{Y}$ & $\mathrm{Y}$ \\
\hline 103 & WL 17, GY 205 & 16.90 & 13.57 & 10.28 & 0.42 & FS & 0.88 & $\mathrm{~N}$ & $\mathrm{~N}$ & $\mathrm{Y}$ ? \\
\hline 108 & EL 29, GY 214 & 17.21 & 12.01 & 7.54 & 0.98 & I & 26. & $\mathrm{~N}$ & $\mathrm{Y}$ & $\mathrm{Y}$ \\
\hline 112 & GY 224 & $>17.00$ & 13.69 & 10.79 & 0.34 & FS & 1.7 & $\mathrm{~N}$ & $\mathrm{~N}$ & $\mathrm{~N} ?$ \\
\hline 118 & IRS 33, GY 236 & $>17.00$ & 15.27 & 12.23 & 0.32 & FS & 0.43 & $\mathrm{~N}$ & $\mathrm{~N}$ & $\mathrm{~N} ?$ \\
\hline 119 & IRS 35, GY 238 & $>17.00$ & 16.25 & 12.73 & 0.30 & FS & 0.45 & $\mathrm{~N}$ & $\mathrm{~N}$ & $\mathrm{~N}$ \\
\hline 121 & WL 20, GY $240 \dagger$ & 13.45 & 10.78 & 9.21 & -0.07 & I & 1.5 & $\mathrm{Y}$ & $\mathrm{N}$ & $\mathrm{Y}$ ? \\
\hline 124 & IRS 37, GY 244 & $>17.00$ & 13.90 & 10.95 & 0.35 & FS & 1.5 & $\mathrm{Y}$ & $\mathrm{Y}$ & $\mathrm{N}$ \\
\hline 127 & GY 245 & 19.00 & 15.43 & 11.98 & 0.17 & FS & 0.13 & $\mathrm{~N}$ & $\mathrm{~N}$ & $\mathrm{~N}$ \\
\hline 129 & WL 3, GY 249 & $>17.00$ & 14.49 & 11.20 & 0.23 & FS & 1.6 & $\mathrm{~N}$ & $\mathrm{Y}$ & $\mathrm{N} ?$ \\
\hline 132 & IRS 42, GY 252 & 15.21 & 11.31 & 8.41 & 0.08 & FS & 5.6 & $\mathrm{~N}$ & $\mathrm{~N}$ & $\mathrm{~N} ?$ \\
\hline 134 & WL 6, GY 254 & $>17.00$ & 14.39 & 10.04 & 0.59 & I & 1.7 & $\mathrm{~N}$ & $\mathrm{~N}$ & $\mathrm{~N}$ \\
\hline 137 & CRBR 85 & $>17.00$ & 17.13 & 13.21 & 1.48 & I & 0.36 & $\mathrm{~N}$ & $\mathrm{~N}$ & $\mathrm{Y}$ \\
\hline 139 & GY 260 & $>17.00$ & 15.71 & 12.54 & -0.03 & FS & 0.30 & $\mathrm{~N}$ & $\mathrm{~N}$ & $\mathrm{~N}$ \\
\hline 141 & IRS 43, GY 265 & $>17.00$ & 13.17 & 9.46 & 0.98 & I & 6.7 & $\mathrm{Y}$ & $\mathrm{Y}$ & $\mathrm{Y}$ \\
\hline 143 & IRS 44, GY 269 & $>17.00$ & 13.09 & 9.65 & 1.57 & I & 8.7 & $\mathrm{~N}$ & Y & $\mathrm{Y}$ \\
\hline 145 & IRS 46, GY 274 & $>17.00$ & 14.65 & 11.46 & 0.94 & I & 0.62 & $\mathrm{~N}$ & $\mathrm{~N}$ & Y? \\
\hline 147 & IRS 47, GY 279 & 15.44 & 11.64 & 8.95 & 0.17 & FS & 3.7 & $\mathrm{~N}$ & $\mathrm{Y}$ & $\mathrm{N}$ \\
\hline 159 & IRS 48, GY 304 & 10.53 & 8.65 & 7.42 & 0.18 & FS & 7.4 & $\mathrm{~N}$ & $\mathrm{~N}$ & $\mathrm{~N}$ \\
\hline 161 & GY 301 & $>17.00$ & 14.77 & 11.30 & 0.12 & FS & 1.8 & $\mathrm{~N}$ & $\mathrm{~N}$ & $\mathrm{~N} ?$ \\
\hline 165 & GY 312 & 16.23 & 13.75 & 11.93 & 0.03 & FS & 0.091 & $\mathrm{~N}$ & $\mathrm{~N}$ & $\mathrm{~N}$ \\
\hline 167 & IRS 51, GY 315 & 17.12 & 12.42 & 8.93 & -0.04 & FS & 1.1 & $\mathrm{Y}$ & $\mathrm{N}$ & Y? \\
\hline 170 & В $162741-244645$ & $>17.00$ & 15.71 & 13.54 & 0.51 & FS & 0.065 & $\mathrm{~N}$ & $\mathrm{~N}$ & $\mathrm{~N}$ \\
\hline 175 & GY 344 & 16.81 & 14.05 & 11.84 & 0.02 & FS & 0.10 & $\mathrm{~N}$ & $\mathrm{Y}$ & $\mathrm{N}$ \\
\hline 182 & IRS 54, GY 378 & 16.63 & 13.50 & 10.87 & 1.76 & I & 6.6 & $\mathrm{Y}$ & $\mathrm{Y}$ & $\mathrm{Y}$ ? \\
\hline 200 & ISO 1631434-245524 & 14.31 & 12.00 & 10.43 & 0.22 & FS & 1.3 & $\mathrm{~N}$ & $\mathrm{~N}$ & $\mathrm{~N}$ \\
\hline 203 & ISO 1631520-245536 & $>18.45$ & 14.86 & 12.73 & 1.19 & I & 1.0 & $\mathrm{~N}$ & $\mathrm{Y}$ & $\mathrm{N} ?$ \\
\hline 209 & IRS 67 & $>17.30$ & 13.31 & 10.43 & 0.75 & I & 1.5 & $\mathrm{Y}$ & $\mathrm{Y}$ & $\mathrm{Y}$ \\
\hline 210 & ISO 1632021-245616 & 15.81 & 14.08 & 13.02 & 0.09 & FS & 0.094 & Y & $\mathrm{N}$ & $\mathrm{N}$ \\
\hline
\end{tabular}

The final images contain two Class I sources and two flat spectrum objects. The image quality was comparable to that of our CFHT images, with a 0.55 FWHM, which enables us to combine this image with our survey.
All images of a given object with the same integration time were median-combined to obtain a sky estimate, which was then subtracted from each individual image. The images were then flat-fielded, aligned based on the location of the 


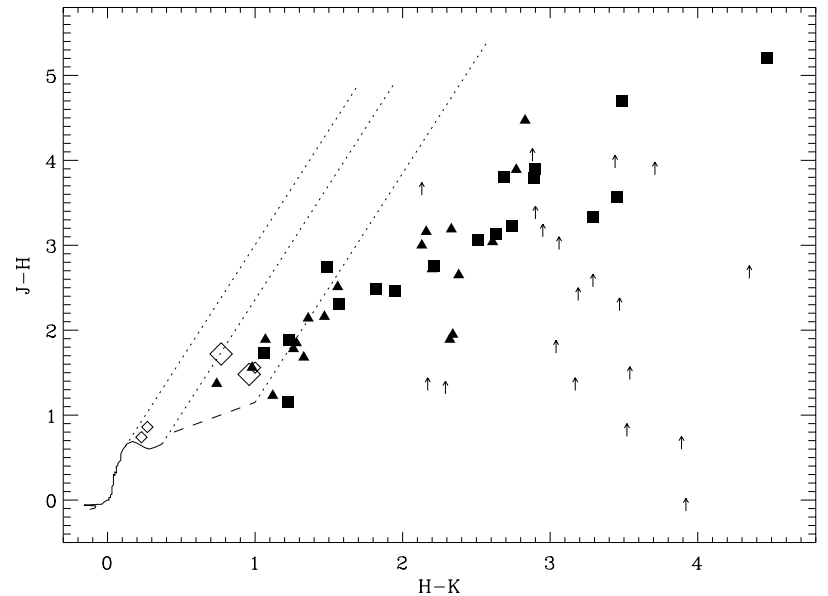

Fig. 1. Near-infrared color-color magnitude for all our targets in Taurus (filled triangles) and Ophiuchus (filled squares). Objects not detected by 2MASS in the $J$ band are indicated with arrows. The companions detected at all three wavelengths in our survey are indicated by diamonds, the larger ones representing those that are likely to be bound to one of our targets. The solid curve indicates the locus of main sequence sources and the dashed line represents the dereddened locus of all T Tauri stars in the Taurus region (Meyer 1996). The dotted lines delineate the reddening band of both loci for $A_{\mathrm{V}}=25 \mathrm{mag}$ and the extinction law from Rieke \& Lebofsky (1985).

brightest unsaturated source in the field, and median combined to produce the final images used in this study. The astrometry of the multiple systems was estimated using the known pixel scale and orientation of the detectors; uncertainties are of order $0.5 \%$ for the plate scale and $1^{\circ}$ for the orientation.

\section{Results}

The objective of our study is to identify physical companions to embedded protostars in Taurus and Ophiuchus. We first identify all visual companions in the $0.8-10^{\prime \prime}$ (110-1400 AU) separation range (Sect. 3.1) and compare our results with those of previous studies of protostars in both areas (Sect. 3.2). We then estimate which of these companions are likely to be merely chance projections of background/foreground objects and finally derive the resulting multiplicity frequency of embedded YSOs in Taurus and Ophiuchus (Sect. 3.3).

\subsection{Visual companions to Class I and flat spectrum sources}

Although embedded sources are located in dense molecular clouds, imaging studies as deep as the one presented here can reveal a large number of faint objects that are mostly unrelated to the star-forming region. We therefore limited the search for companions to within a 10" (1400 AU) radius around the surveyed embedded YSOs, a cutoff which excludes the presence of many background stars projected by chance in the vicinity of our targets (see Sect. 3.3). Using this search radius also allows us to compare directly our results to multiplicity surveys among the more evolved T Tauri stars, which have been conducted over the same separation range. Furthermore, if the distribution of orbital periods of protostellar multiple systems does not evolve significantly with time (e.g., Kroupa \& Burkert 2001), we expect that this separation range will encompass a large fraction of all binary systems, as is the case for main sequence field stars: Duquennoy \& Mayor (1991) have for instance shown that less than $10 \%$ of solar-type field stars have a companion at separations wider than 1400 AU. The lower limit on measurable binary separation is fixed by the sharpness of the images. Systems tighter than the image's FWHM would only be slightly elongated and could be misidentified as wind shaking or telescope tracking errors. In order to exclude binaries that are only marginally resolved, we do not consider systems tighter than 0.'8 (110 AU). Tighter systems will be the focus of a higher resolution study of the same sources performed with adaptive optics (Duchêne et al., in prep.).

The $1400 \mathrm{AU}$ upper cutoff adopted here is several times smaller than the size of individual prestellar cores in both starforming regions (Motte et al. 1998; Motte \& André 2001). Therefore, two stars physically separated by such a "small" distance most likely formed through the fragmentation of a single core. However, it has been shown that the typical size of molecular cores differs in the two areas, with cores in Taurus being $\sim 3$ times larger than those in Ophiuchus (see Sect. 1). In order to study the core fragmentation process, it might be more relevant to search for binaries using a similar separation cutoff relative to the original core size. Therefore, we have also considered candidate companions up to 30" (4200 AU) from our YSO targets in Taurus, i.e., less than a third of the size of a typical prestellar core in that region. In our analysis, we use the $10^{\prime \prime}$ cutoff to define our primary sample of companions. The enlarged separation range probed in Taurus is only used for discussing core size-scaled binary properties in both star-forming regions (see Sect. 4.1).

In the $0 .{ }^{\prime} 8-10^{\prime \prime}$ range, we found a possible companion to five of the 22 protostars we surveyed in Taurus, while in Ophiuchus, we found 14 candidate companions associated to 11 targets in our sample of 41 protostars. The observed raw companion star fractions ${ }^{1}$ are $23 \pm 9 \%$ in Taurus and $34 \pm 7 \%$ in Ophiuchus. In the 10-30" range around our Taurus targets we find 19 additional possible companions, although a majority of them is likely to be projected background stars (see Sect. 3.3). Images of the possible higher-order multiple systems with separations $\leq 10^{\prime \prime}$ are presented in Fig. 2 . The astrometric and photometric properties of all candidate companions are summarized in Table 3 . The faintest two companions to IRS 54 were detected by combining a short integration image with a much longer exposure of the same field; they are therefore excluded from our discussion as we obtained such deep images for only a handful of objects in our sample.

\footnotetext{
1 Throughout this study we adopt the binomial statistics for estimating uncertainties associated with the companion star fraction, $\sigma_{\mathrm{CSF}}=\sqrt{N_{\text {comp }}\left(1-N_{\text {comp }} / N_{\text {prim }}\right)} / N_{\text {prim }}$ (where $N_{\text {prim }}$ and $N_{\text {comp }}$ are the number of primaries and companions, respectively), as a more appropriate method for this problem than the widely used Poisson statistics since the $N_{\text {comp }} / N_{\text {prim }} \ll 1$ criteria is usually not met.
} 


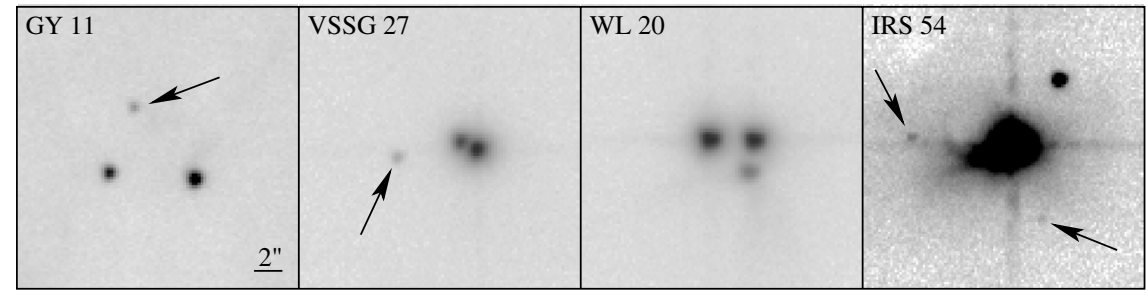

Fig. 2. Greyscale images of the candidate multiple systems detected in Ophiuchus. Each image is about $20^{\prime \prime}$ (2800 AU) on a side; North is up and East to the left. The faintest companions are indicated with arrows.

Table 3. List of all companions detected within 10" (Ophiuchus) or 30" (Taurus) of our targets. Flux ratios are measured with respect to the brightest component in the $K$ band image, except for three systems where the Class I source is the faintest component in the near-infrared († symbol). The boldfaced entries represent systems that are likely to be physically bound (see Sect. 3.3). The two companions to IRS 54 listed in italics were detected by combining a short and a long exposure and are not included in our survey. Upper limits for flux ratios are at the $3 \sigma$ level. IRAS $04264+2433$ was saturated in all of our images, so only a lower limit for the flux ratio to its companion can be obtained.

\begin{tabular}{|c|c|c|c|c|c|c|c|}
\hline Object & $\begin{array}{c}\rho \\
\left({ }^{\prime \prime}\right)\end{array}$ & $\begin{array}{l}\text { PA } \\
\left({ }^{\circ}\right)\end{array}$ & $\begin{array}{c}\Delta J \\
(\mathrm{mag})\end{array}$ & $\begin{array}{c}\Delta H \\
(\mathrm{mag})\end{array}$ & $\begin{array}{c}\Delta K \\
(\mathrm{mag})\end{array}$ & $\begin{array}{c}\Sigma\left(K \leq K_{\mathrm{B}}\right) \\
\left(10^{-4} \operatorname{arcsec}^{-2}\right)\end{array}$ & $\overline{P_{\text {bound }}}$ \\
\hline \multicolumn{8}{|c|}{ Taurus $\left(0 .{ }^{\prime} 8<\rho<30^{\prime \prime}\right)$} \\
\hline IRAS 04016+2610 & 23.28 & 223.5 & & & $4.60 \pm 0.05$ & 1.6 & 0.76 \\
\hline IRAS 04108+2803 B & 22.16 & 237.1 & & & $0.17 \pm 0.02$ & 0.4 & 0.94 \\
\hline- & 15.23 & 274.1 & & & $6.00 \pm 0.05$ & 1.2 & 0.92 \\
\hline IRAS 04113+2758 & 4.03 & 152.9 & $-0.94 \pm 0.03$ & $-0.28 \pm 0.03$ & $0.12 \pm 0.03$ & 0.8 & 0.99 \\
\hline- & 24.80 & 279.8 & $2.96 \pm 0.05$ & $3.54 \pm 0.05$ & $3.90 \pm 0.05$ & 1.6 & 0.73 \\
\hline IRAS $04158+2805$ & 25.45 & 27.3 & $-0.44 \pm 0.02$ & $0.05 \pm 0.02$ & $0.94 \pm 0.02$ & 0.8 & 0.85 \\
\hline- & 28.95 & 246.5 & $3.86 \pm 0.05$ & $4.23 \pm 0.05$ & $5.08 \pm 0.05$ & 5.3 & 0.25 \\
\hline IRAS 04181+2654 B & 22.22 & 248.5 & & & $4.45 \pm 0.05$ & 2.5 & 0.68 \\
\hline IRAS 04191+1523 & 6.09 & 303.7 & $>2.05$ & $2.15 \pm 0.05$ & $2.06 \pm 0.03$ & 0.8 & 0.99 \\
\hline- & 28.30 & 290.0 & $>2.05$ & $>4.50$ & $4.80 \pm 0.10$ & 6.5 & 0.20 \\
\hline IRAS 04239+2436 & 22.12 & 319.9 & & & $4.80 \pm 0.05$ & 1.2 & 0.83 \\
\hline IRAS 04248+2612 & 4.55 & 15.1 & & & $4.60 \pm 0.10$ & 3.7 & 0.98 \\
\hline IRAS 04260+2642 & 12.30 & 279.4 & & & $5.67 \pm 0.10$ & 9.0 & 0.65 \\
\hline IRAS 04263+2426 & 1.27 & 353.3 & & & $1.05 \pm 0.05$ & 0.4 & 0.99 \\
\hline IRAS $04264+2433$ & 10.93 & 295.6 & $>6.1$ & $>6.4$ & $>5.9$ & 2.5 & 0.91 \\
\hline IRAS $04302+2247$ & 22.64 & 120.5 & & & $3.88 \pm 0.05$ & 5.3 & 0.43 \\
\hline- & 27.27 & 263.2 & & & $4.20 \pm 0.05$ & 7.8 & 0.16 \\
\hline- & 19.34 & 114.4 & & & $4.40 \pm 0.08$ & 8.6 & 0.37 \\
\hline IRAS $04325+2402$ & 8.15 & 350.5 & & $2.42 \pm 0.10$ & $2.43 \pm 0.05$ & 1.2 & 0.98 \\
\hline IRAS $04365+2535$ & 12.48 & 133.6 & & & $6.25 \pm 0.15$ & 5.7 & 0.76 \\
\hline IRAS $04381+2540$ & 25.92 & 177.4 & & & $6.80 \pm 0.15$ & 5.3 & 0.33 \\
\hline IRAS $04385+2550$ & 18.90 & 342.0 & $2.73 \pm 0.03$ & $2.90 \pm 0.03$ & $3.20 \pm 0.03$ & 0.4 & 0.96 \\
\hline IRAS 04889+3042 & 12.32 & 20.5 & & & $4.46 \pm 0.05$ & 2.4 & 0.89 \\
\hline- & 20.67 & 295.9 & & & $6.05 \pm 0.10$ & 6.9 & 0.40 \\
\hline \multicolumn{8}{|c|}{ Ophiuchus $\left(0{ }^{\prime} .8<\rho<10^{\prime \prime}\right)$} \\
\hline LFAM 1 $\dagger$ & 9.35 & 104.9 & & & $-4.03 \pm 0.10$ & 1.1 & 0.97 \\
\hline GY 11 & 6.20 & 86.1 & & & $0.76 \pm 0.05$ & 3.2 & 0.96 \\
\hline- & 6.73 & 40.9 & & & $2.10 \pm 0.10$ & 4.5 & 0.94 \\
\hline VSSG 27 & 1.22 & 64.3 & & $\mathbf{0 . 8 4} \pm \mathbf{0 . 1 0}$ & $1.32 \pm 0.05$ & 0.7 & 0.99 \\
\hline- & 5.73 & 95.7 & & $4.81 \pm 0.15$ & $5.29 \pm 0.05$ & 2.4 & 0.98 \\
\hline WL 2 & 4.24 & 341.1 & & $1.35 \pm \mathbf{0 . 0 3}$ & $1.36 \pm 0.02$ & 0.4 & 0.99 \\
\hline WL $20 \dagger$ & 3.66 & 51.5 & & & $-3.13 \pm 0.03$ & 0.2 & 0.99 \\
\hline- & 2.24 & 351.9 & & & $-2.89 \pm 0.03$ & 0.2 & 0.99 \\
\hline $\operatorname{IRS} 37 \dagger$ & 8.68 & 63.7 & & & $-1.60 \pm 0.03$ & 0.2 & 0.99 \\
\hline IRS 43 & 7.12 & 321.5 & & & $2.98 \pm 0.05$ & 1.3 & 0.98 \\
\hline IRS 51 & 1.62 & 9.0 & & & $3.67 \pm 0.05$ & 1.1 & 0.99 \\
\hline IRS 54 & 7.17 & 323.1 & & $5.40 \pm 0.10$ & $5.10 \pm 0.03$ & 1.9 & 0.97 \\
\hline- & 9.01 & 86.4 & & $>5.9$ & $7.97 \pm 0.05$ & 7.8 & 0.82 \\
\hline- & 7.14 & 202.4 & & $>5.9$ & $9.09 \pm 0.10$ & 2.0 & 0.73 \\
\hline IRS 67 & 2.83 & 300.0 & & & $4.13 \pm 0.10$ & 9.1 & 0.98 \\
\hline ISO $1632021-245616$ & 7.78 & 179.8 & & & $6.7 \pm 0.2$ & 15. & 0.75 \\
\hline
\end{tabular}


Our ability to detect a companion depends on its flux relative to its brighter primary: the closer the companion, the brighter it needs to be for us to detect it. This is illustrated in Fig. 3, where we have plotted all observed companions in our images. We have also included in this figure companions to well-exposed Ophiuchus T Tauri multiple systems that lie within our field of view (see Appendix A), in order to better define the lower envelope of all detected companions. It appears that binaries with $\Delta K \leq 7-8$ mag can be detected beyond 6 " or so, while at $1^{\prime \prime}$ only binaries with $\Delta K \leq 3$ mag are identified. Separately, we have estimated our ability to detect faint point sources around bright sources by computing the standard deviation of pixel values in concentric annuli surrounding single sources. The resulting average $3 \sigma$ limit is also plotted in Fig. 3 along with the standard deviation (from observations of 16 independent point sources) about that curve. These curves match relatively well the lower envelope of our detected companions. Within the central 2-3", the detection limit mostly depends on the seeing but it does not appear to vary dramatically from a source to another. At larger distances, the detection limit is mostly driven by the background noise, which is roughly constant except if the protostar is surrounded by some extended nebulosity.

We conclude that we have detected all possible companions that lie above the solid curve in Fig. 3. For objects that have an extended nebulosity in our near-infrared images, our ability to detect a nearby companion can be somewhat impaired. As illustrated in Fig. 4, nebulosities range from a faint extended halo (e.g., IRAS 04169+2702) to the two parallel nebulae associated with the presence of an edge-on, optically thick circumstellar disk that blocks the direct starlight (IRAS 04302+2247). However, only extremely bright nebulosities in which no pointlike object can be seen, such as IRAS $04302+2247$, significantly affect our detection limit. Indeed, the fraction of visual companions we detected around objects with an infrared nebulosity ( 9 out of $24,38 \pm 10 \%$ ) is similar to that observed around point like sources ( 10 out of $39,26 \pm 7 \%$ ). We conclude that we probably did not miss many companions due to the presence of extended nebulosities over the separation range investigated in this study.

\subsection{Comparison to previous surveys}

In the Taurus-Auriga molecular cloud, this study is the first census of multiple systems among a nearly complete sample of embedded YSOs. We note that IRAS $04113+2758$ is a resolved pair in 2MASS, while the binarity of IRAS $04263+2426$ (=Haro 6-10) was discovered by Leinert \& Haas (1989). The $\sim 6^{\prime \prime}$ companion to IRAS $04191+1523$ has also been detected in the millimeter continuum with the IRAM Plateau de Bure Interferometer, confirming that both sources are young stellar objects with compact envelopes and/or disks (Motte 1998). Finally, our images reveal an almost point-like object located about 3" North of IRAS $04016+2610$. Higher spatial resolution images of this system by Padgett et al. (1999) show an extended structure, most likely to be light scattered off the envelope or disk associated to this YSO. We therefore reject this

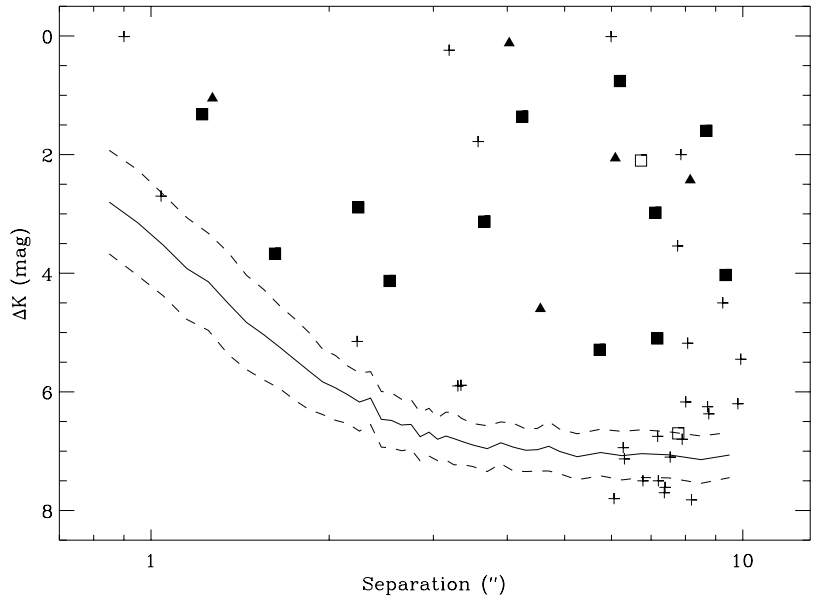

Fig. 3. Observed flux ratios of our targets (Taurus: triangles; Ophiuchus: squares) and of Class II and III systems in Ophiuchus (plus signs). The filled and empty symbols for our Ophiuchus targets discrimate between physically bound and projected systems (see Sect. 3.3). The solid line is our median $3 \sigma$ detection limit, and the dashed lines represent the $1 \sigma$ variation about that line (see text for details).

"companion" from our analysis. Of the five companions to embedded sources in Taurus reported here, two are new detections (IRAS 04248+2612, IRAS 04325+2402).

Some of the widest pairs we identified in Ophiuchus were already known: LFAM 1 (whose companion is GY $12 \equiv$ ISO 34), IRS 43 (its companion is GY 263), WL 2 (from 2MASS), WL 20 (Ressler \& Barsony 2001), GY 11 and IRS 37 (Allen et al. 2002). In their recent survey of embedded YSOs, Haisch et al. (2004) further identified the companions to IRS 54 and VSSG 27. The separations and position angle measurements for these systems agree well with their previously published values. We further report here the detection of companions to two targets that were also investigated by Haisch et al. (2002), namely IRS 51 and IRS 67. Haisch et al. (2002) detected our proposed companion to IRS 51 but their nondetection of the secondary at $10 \mu \mathrm{m}$ led them to conclude that it was not real but rather a patch of scattered light in a possible outflow. The companion appears indistinguishable from a point source at the 0.' 6 resolution of our image of the system. We therefore believe that the companion is real and consider that its non-detection at $10 \mu \mathrm{m}$ results from the limited signal-to-noise ratio of the image of Haisch et al. (their Fig. 1). The companion to IRS 67 was probably not detected by Haisch et al. because of its small separation, 2". 5 , and large flux ratio, $\Delta K \sim 4.1 \mathrm{mag}$. Overall, of the 14 companions we report here for embedded sources in Ophiuchus, three are new detections (IRS 51, IRS 67 and ISO 1632021-245616).

Overall, our survey thus revealed five new companions in the $0{ }^{\prime} 8-10^{\prime \prime}$ separation range, as well as the first infrared detection of a companion only detected at millimeter wavelengths so far. This significantly adds to the statistics of multiplicity of embedded YSOs in both star-forming regions. Haisch et al. (2004) recently surveyed 6 star-forming regions, with YSO samples ranging from 9 to 30 objects per region, and derived an overall companion star fraction of 12/76 (16 $\pm 4 \%)$ 


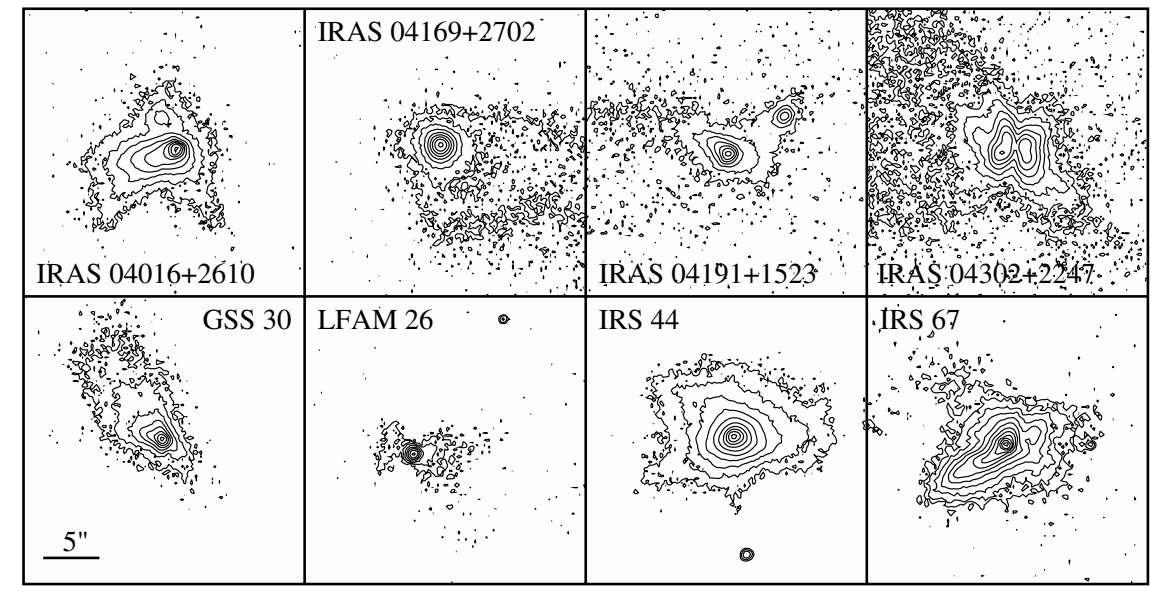

Fig. 4. Example of nebulosities surrounding some of our targets; the upper row shows some Taurus sources, the lower Ophiuchus targets. Each image is $25^{\prime \prime}$ on the side; North is up and East to the left. The contours are spaced by $0.75 \mathrm{mag} / \mathrm{arcsec}^{2}$ from the peak flux down to approximately the $4-5 \sigma$ limit. over the separation range 300-1400 AU and down to a flux ratio $\Delta K=4.0 \mathrm{mag}$. Over the same separation range and detection limits, we derive a companion star fraction of 13/63 (20 \pm $5 \%$ ) for embedded YSOs in Taurus and Ophiuchus. The two surveys yield very similar results, even though the former includes smaller samples of YSOs distributed over a number of star-forming regions, while our survey deals with nearly complete YSO populations in only two star-forming regions. The similar results obtained from the two surveys thus suggests that the average companion star fraction for embedded sources does not vary much from one star-forming region to the other. Using high resolution radio continuum imaging, Reipurth et al. (1999, 2002 , 2004) have compiled a list of 21 embedded protostars in several star-forming regions that includes four multiple systems with a separation in the range 110-1400 AU studied here, a multiplicity rate also very similar to the one we find here.

\subsection{Physically bound multiple systems}

The candidate companions we have identified could be either physically bound to our targets or unrelated objects (YSOs or field stars) that are projected within $10^{\prime \prime}$ (or 30"). Since we only have $K$ band photometry for most of our candidate companions, it usually is not possible to use color indices to determine their nature. The two visual companions to IRAS $04158+2805$ are likely background stars, given their location in the nearinfrared color-color diagram (see Fig. 1). In any case, the large and variable extinction across the molecular clouds would represent a major issue, as a much redder companion could be interpreted both as an extincted background star or a more deeply embedded companion protostar.

We therefore used a statistical approach to estimate the probability that the companions we found are physically bound to their primary. Our method relies on the large field of view of our images, which allows us to estimate the local surface density of faint objects in the field. More specifically, we used the same method as described in Duchêne et al. (2001), which we summarize here briefly.

We first count the number of objects located in our field of view that are at least as bright as the candidate companion, including the companion itself but not its primary.
We convert this number into the average surface density of objects brighter than this threshold, $\Sigma\left(K \leq K_{\mathrm{B}}\right)$. The median value of this quantity over all fields is about $2 \times 10^{-4} \mathrm{arcsec}^{-2}$. Assuming a random distribution of background stars across the fields, we then estimated the number of companions one would expect to lie as close to the primary as the companion or closer. This number is $\bar{N}=\pi\left(\rho_{\mathrm{B}}^{2}-\rho_{\text {lim }}^{2}\right) \times \Sigma\left(K \leq K_{\mathrm{B}}\right)$, where $\rho_{\mathrm{B}}$ is the binary separation and $\rho_{\text {lim }}=0 . ' 8$ is the smallest separation probed in this survey. Finally, we estimated the probability that a random uniform distribution of stars across the field yields no companion within $\rho_{\mathrm{B}}$, which we estimate using Poisson statistics (justified by the fact that $\bar{N}$ is almost always smaller than unity): $P_{\text {bound }}=P(N=0)=\mathrm{e}^{-\bar{N}}$. The resulting probabilities of the companion being physically associated to a YSO are given in the last column of Table 3 . By construction, this method takes into account the projection of background and foreground objects, as well as of unrelated cloud members.

Among the $0 .{ }^{\prime} 8-10^{\prime \prime}$ companions detected in our survey, five out of 14 in Ophiuchus and three out of five in Taurus have probabilities of being bound larger than $99 \%$. These are very likely physical systems but we have to decide on a threshold down to which we accept the companions as likely. On the other hand, most Taurus companions between $10^{\prime \prime}$ and 30" have probabilities lower than $90 \%$ of being bound. These are likely to be projected unrelated stars. In the following, we decided to consider only systems that have probabilities of being bound larger than $95.5 \%$, i.e., for which a background status can be rejected at the $2 \sigma$ level. This is probably a conservative estimate, as our method explicitly assumes that background stars are randomly located in the fields while our images show that the protostars are usually found in darker areas, with an increasing number of sources towards the edges of our images (i.e., YSOs are located deeper inside molecular clouds). This is however an image-to-image effect whose amplitude is difficult to estimate because of the relatively small number of stars per field. On the other hand, members of young associations tend to cluster in relatively compact groups, which may produce apparent pairs of YSOs that are in fact unbound. For instance, among the companions retained with this criterion, one (GY 12, companion of LFAM 1) is a known member of the Ophiuchus star-forming region (see Sect. 3.2) that appears to be 
a Class III, weak-line T Tauri star. It is not clear whether two objects that have such a different evolutionary status do form a physically bound binary system or whether this is the mere result of the projection of two members within $10^{\prime \prime}$. In any case, background contamination can only be estimated on a statistical basis and there is no single threshold that perfectly separates physical systems from projected ones. With this caveat in mind, we adopt the $2 \sigma$ threshold in our analysis.

In our survey, we therefore have identified five binary systems with separation $0.8<\rho<10^{\prime \prime}$ out of 22 protostars in Taurus and 12 companions to 10 of our 41 sources in Ophiuchus. This corresponds to a companion star fraction of $23 \pm 9 \%$ in Taurus, $29 \pm 7 \%$ in Ophiuchus and $27 \pm 6 \%$ in the 110-1400 AU range for the combined sample. Our detection limit is not uniform across the separation range probed in this study and we may therefore be missing a few close, faint companions to our targets. We do not attempt to correct for this, as one would have to rely on assumptions regarding the flux ratio and separation distributions of protostellar binary systems, which are currently unknown. Consequently, the number of companions we estimate might slightly underestimate the actual one over the separation range investigated here. In addition, we find one candidate companion in Taurus which is possibly physically bound, though just at the $2 \sigma$ level, to a YSO in the range $10-30^{\prime \prime}$.

\section{Discussion}

The survey we have conducted yields a statistically significant estimate of the multiplicity frequency for a nearly complete population of embedded YSOs in Taurus and Ophiuchus. We discuss below the implications of this result regarding the fragmentation of pre-stellar cores as they collapse (Sect. 4.1). We then investigate whether prompt dynamical evolution of multiple systems occurs during the protostellar embedded stage, on a timescale of $\gtrsim 10^{5} \mathrm{yr}$, by comparing the statistics of multiplicity we derived for Class I and flat spectrum sources to that of even younger, more deeply embedded Class 0 sources. We further compare the frequency of embedded multiple systems to that of Class II and III T Tauri stars in the same clouds, in order to investigate the dynamical evolution of multiple systems on a longer timescale of $\gtrsim 10^{6} \mathrm{yr}$ (Sect. 4.2). Finally, we confront the predictions of various classes of models dealing with the formation and early evolution of multiple stellar systems to the statistics of multiplicity of embedded sources (Sect. 4.3).

\subsection{Multiple protostellar systems and the fragmentation of collapsing cores}

The primary result of this study is that, over the separation range 110-1400 AU, embedded YSOs present similar apparent multiplicity rates in the Taurus and Ophiuchus star-forming regions despite the known differences between these two regions (pre-stellar core properties and stellar density). The size of prestellar cores being $\sim 3$ times larger in Taurus than in Ophiuchus, the comparison of the properties of binary systems spanning the same physical separation range may introduce a bias if core fragmentation is a scale-free process (e.g., Sterzik et al. 2003). We therefore also compare the 110-1400 AU multiplicity rate of Ophiuchus $(29 \pm 7 \%)$ to that over the 330-4200 AU separation range in Taurus. The latter is $23 \pm$ $9 \%$ (five companions out of 22 targets), statistically indistinguishable from the Ophiuchus rate. This alternative comparison reinforces our conclusion that the frequency of companions among our two samples of embedded YSOs is identical in the two star-forming regions. This in turn suggests that, in spite of initial differences between Taurus and Ophiuchus pre-stellar cores, fragmentation appears to produce very similar properties for their multiple systems at the protostellar stage.

Both samples of embedded YSOs we studied are nearly complete. Nevertheless, they exhibit a notable difference: roughly $25 \%$ of the primaries in Ophiuchus have $L_{\mathrm{bol}} \leq 0.1 L_{\odot}$, while only one such source is included in the Taurus sample. These low luminosity objects may be either extremely low mass objects (e.g. candidate embedded brown dwarfs) or embedded sources with reduced accretion rates. In any event, it might not be fair to compare the multiplicity properties of these two samples if they are made up of intrinsically different populations (different mass function and/or evolutionary stages). The median luminosity of both samples is about $0.5 L_{\odot}$. We compared the multiplicity rate of the faintest half of our samples to that of the brightest half. Overall, we find a marginally higher multiplicity rate among objects with $L_{\text {bol }}>0.5 L_{\odot}$ (11 companions to 33 sources) than among less luminous ones (6 out of 30), with both molecular clouds suggesting the same trend although not at a statisticaly significant level due to the small sample sizes. Hence, irrespective of whether we include all embedded YSOs in our sample or not, the overall companion star fraction over the 110-1400 AU range is of order 20-30\% in both star-forming regions.

The observed proportion of companions to protostars we find is relatively high, especially if one considers that hardly more than an order magnitude in separation is probed in this study. In the same separation range, the companion star fraction of field G dwarfs amounts to only 14\% (Duquennoy \& Mayor 1991). This comparison is relevant if the distribution of separation for protostellar systems is as wide as that for mature systems. Kroupa \& Burkert (2001) concluded from $N$-body simulations that stellar dynamical interactions in embedded clusters cannot significantly broaden the initial distribution of orbital periods, so that the wide range of separations observed for mature systems must result from the fragmentation process itself. At an earlier stage, accretion of material from the infalling envelope on a "seed" protobinary may also change the initial separation of the binary. Through SPH simulations, Bate (2000) has shown that this phenomenon is largely dependent on the (unknown) initial conditions. While we may expect accreting binary systems to get wider with time on average, we note that the excess of close companions in the separation range studied here is almost twice as large as the frequency of systems with larger separations for field $\mathrm{G}$ dwarfs. Hence, the excess companion star fraction we find over a restricted range of separation in embedded sources suggests that binary and multiple systems are indeed a very frequent outcome of the dynamical fragmentation of prestellar cores as they collapse. 
Table 4. Observed companion star fraction over the separation range 110-1400 AU in Perseus, Taurus-Auriga and Ophiuchus star-forming regions (SFRs) for Class 0 sources, Class I sources with (env+) and without (env-) an extended millimetric envelope, and for Class II-III T Tauri stars in the same clouds. For comparison, the multiplicity rate for G-type field dwarfs over the same separation range is $14 \%$ (Duquennoy \& Mayor 1991). References: 1 - Looney et al. (2000); 2 - this study; 3 - Mathieu (1994); 4 - Barsony et al. (2003).

\begin{tabular}{|c|c|c|c|c|c|c|c|}
\hline & SFR & $N_{\text {comp }} / N_{\text {prim }}$ & $\begin{array}{l}\text { CSF } \\
(\%)\end{array}$ & Ref. & $\begin{array}{c}\mathrm{CSF}_{\text {tot }} \\
(\%)\end{array}$ & $\begin{array}{c}\mathrm{CSF}_{\text {tot }} \\
(\%)\end{array}$ & \\
\hline Class 0 & $\begin{array}{c}\text { Perseus } \\
\text { Ophiuchus }\end{array}$ & $\begin{array}{l}1 / 11 \\
2 / 2 \\
\end{array}$ & $25 \pm 13$ & 1 & $25 \pm 13$ & \multirow{2}{*}{$38 \pm 8$} & \multirow{2}{*}{$\begin{array}{l}\text { Embedded sources } \\
\text { with mm envelope }\end{array}$} \\
\hline Class I env+ & $\begin{array}{c}\text { Taurus } \\
\text { Ophiuchus }\end{array}$ & $\begin{array}{l}5 / 12 \\
7 / 15\end{array}$ & $\begin{array}{l}41 \pm 14 \\
47 \pm 13\end{array}$ & 2 & $44 \pm 10$ & & \\
\hline Class I env- & $\begin{array}{c}\text { Taurus } \\
\text { Ophiuchus }\end{array}$ & $\begin{array}{l}0 / 10 \\
5 / 26\end{array}$ & $\begin{array}{c}0 \\
19 \pm 8\end{array}$ & 2 & $14 \pm 6$ & \multirow{2}{*}{$22 \pm 3$} & \multirow{2}{*}{$\begin{array}{l}\text { Optical/IR sources } \\
\text { without mm envelope }\end{array}$} \\
\hline Class II-III & $\begin{array}{c}\text { Taurus } \\
\text { Ophiuchus }\end{array}$ & $\begin{array}{c}24 / 100 \\
21 / 88\end{array}$ & $\begin{array}{l}24 \pm 4 \\
24 \pm 5\end{array}$ & $\begin{array}{l}3 \\
4\end{array}$ & $24 \pm 3$ & & \\
\hline
\end{tabular}

The similitudes of the multiple systems in the two molecular clouds extend beyond their frequency, and also include as well their separation distribution and $K$ band flux ratios, which both evenly sample the ranges probed in this survey (Fig. 3). Protostellar multiple systems appear to span a larger range of flux ratios than the more evolved $\mathrm{T}$ Tauri (Class II) binaries, which are usually limited to $\Delta K<3$ mag (e.g., Leinert et al. 1993). This may be because the observed $K$ band flux of an embedded YSO is mostly driven by its accretion rate and line of sight extinction, while the central source's luminosity is usually a small fraction of the total system's luminosity (Bontemps et al. 2001). This is not the case for T Tauri stars, in which near infrared flux ratios more reliably trace mass ratios. Therefore, we cannot use the distribution of flux ratios of our sample of YSOs to infer the underlying mass ratio distribution for these objects, but can merely conclude that the relative accretion rates onto the components of the multiple protostellar systems appear similar in Taurus and in Ophiuchus, in spite of initially different prestellar core structures.

\subsection{The evolution of protostellar multiple systems}

The frequency and orbital properties of protostellar systems are expected to evolve on various timescales due to a number of processes, such as dynamical decay of initially unstable configurations ( $\sim 10^{4-5} \mathrm{yr}$, e.g., Delgado-Donate et al. 2003; Goodwin et al. 2004a) or disruptive encounters with other systems if located in dense environments $\left(\sim 10^{5-6} \mathrm{yr}\right)$. In this context, a somewhat surprising result is the similar frequency and properties we find for protostellar systems in both Taurus and Ophiuchus. This suggests that their early dynamical evolution $\left(\leq 10^{5} \mathrm{yr}\right)$ has been remarkably similar, in spite of both different initial pre-stellar core structures and different environmental conditions, the stellar density in Ophiuchus being two to three orders of magnitude larger than in Taurus (e.g., Allen et al. 2002).
Searching for possible evolutionary effects during the embedded phase, we first separate Class I from flat spectrum (FS) sources within our sample, as the latter are believed to be in an evolutionary state intermediate between Class I and T Tauri objects, at least on a statistical basis. Combining the two starforming regions in order to avoid small sample sizes, we find 9 companions among 28 Class I sources and 8 companions among 35 flat spectrum primaries. This yields a companion star fraction of $32 \pm 9 \%$ for Class I and of $23 \pm 7 \%$, for FS sources over the 110-1400 AU separation range, a hardly significant evolutionary trend.

Our source selection is, however, exclusively based on the near- to mid- infrared spectral index, which may not be the most appropriate tracer of a YSO's evolutionary status. For instance, T Tauri stars that are observed with an optically thick edge-on disk (such as IRAS $04158+2805$ in our sample) can have a spectral index typical of a Class I source. A more reliable indicator of the evolutionary status of an embedded YSO is the presence of an extended envelope, which characterizes a bona fide protostar. Such a protostellar envelope can be directly traced by its thermal millimeter continuum emission (e.g. André \& Montmerle 1994; Motte \& André 2001) and often indirectly traced by an extended near-IR nebulosity (Park \& Kenyon 2002 and this study). The presence/absence of such an envelope is listed for all sources in our sample in Tables 1 and 2. Twelve out of 22 YSOs in Taurus and 15 out of 41 YSOs of Ophiuchus exhibit evidence for a protostellar envelope. Combining the two regions, we find 12 companions to the 27 true protostars surveyed giving a companion star fraction of $44 \pm 10 \%$ in the 110-1400 AU range (see Table 4). In contrast, the more evolved sources in our sample, which have already dissipated their envelope, have a much lower companion star fraction: $14 \pm 6 \%$ (see Table 4). Hence, adopting the presence of an envelope as a qualitative diagnostic of youth, we find that young self-embedded sources tend to be more often in multiple systems than more evolved ones. This evolution of the multiplicity rate, seen here at the $2.6 \sigma$ level, suggests 
a significant reduction of the number of wide multiple systems over a timescale of $\sim 10^{5} \mathrm{y}$, which could conceivably result either from disruptive encounters with other systems or from the internal decay of initially unstable systems.

If this trend can be extrapolated back to even earlier stages of evolution, one may expect to find an even larger fraction of multiple systems among Class 0 sources. The investigation of 14 YSOs driving large scale outflows led Reipurth (2000) to claim a multiplicity frequency in the range $79-86 \%$, much higher than found here for Class I and flat spectrum sources. However, Reipurth's (2000) sample contains sources that span the full range from Class 0 protostars to $\mathrm{T}$ Tauri stars and some of the multiple systems have separations outside the range studied here. Moreover, if giant Herbig-Haro flows are actually triggered by the decay of unstable protostellar multiple systems, as Reipurth (2000) argues, this sample is obviously strongly biased towards the detection of such systems. Therefore, the derived multiplicity frequency of $\sim 80 \%$ probably does not represent the true fraction of multiple systems in deeply embedded YSOs. A more meaningful comparison can be made with the results of the $2.7 \mathrm{~mm}$ continuum interferometric survey of embedded YSOs performed by Looney et al. (2000) with a spatial resolution similar to ours. Their sample contains 12 "separate envelope" Class 0 sources (see their Table 5, assuming that the members of the SVS13 group are all Class 0 objects and classifying SSV13B as a separate envelope source), i.e., sources which would be considered as distinct primaries in our study. Among these, they find three companions in the separation range 110-1400 AU corresponding to their definition of "common envelope systems", to which their millimeter interferometric observations are mostly sensitive. In this range, the companion star fraction of their Class 0 sample is thus $3 / 12=25 \pm$ $13 \%$, including two companions to two sources in Ophiuchus. Such a fraction is consistent with the multiplicity rate we derive for Taurus Class I sources with extended envelopes (see Table 4). Unfortunately, the poor statistics stemming from the small sizes of the samples and the differing separation sensitivities of the millimeter interferometric survey and the present near-IR infrared survey prevent us from drawing any strong conclusion about the evolution of the multiplicity rate between Class 0 and Class I sources on a timescale $<10^{5} \mathrm{yr}$.

Looking further downstream in the evolution of young multiple systems, we can also compare the multiplicity frequency we derived for embedded sources in Taurus and Ophiuchus to the fraction of multiple systems found among more evolved Class II and Class III $\mathrm{T}$ Tauri stars in the same regions. For the latter groups, we used the lists of multiple systems from Mathieu (1994) in Taurus and Barsony et al. (2003) in Ophiuchus, which likely contain most $0 .{ }^{\prime} 8-10^{\prime \prime}$ companions, as those can be found from direct infrared imaging. Beyond $1^{\prime \prime}$, those surveys are likely to be complete and they actually contain systems with flux ratios as large as 4 mag at least, thus similar to our detection limits. In the separation range considered here, there are 21 companions to 88 optically revealed T Tauri stars in Ophiuchus and 24 companions to 100 primaries in Taurus (see Fig. 4). The two populations have a companion star fraction of $24 \pm 3 \%$, i.e., a factor of about two lower than that of Class I sources with envelopes $(44 \pm 10 \%)$. The much lower multiplicity fraction of Class II-III sources in the two clouds compared to that of the youngest Class I sources surrounded by millimeter envelopes (a $1.9 \sigma$ difference) seems to confirm the similar trend reported above between young and evolved embedded Class I sources.

Table 4 summarizes the statistics of multiplicity among embedded sources and young PMS stars in Taurus and Ophiuchus. The most significant result, yet still relying on relatively small samples, is provided by the comparison between deeply embedded Class 0 and Class I sources surrounded by extended millimeter envelopes on the one hand, and more evolved, envelope-less Class I-III sources, on the other. The companion star fraction is seen to decrease from $38 \pm 8 \%$ in the youngest embedded sources (age $\lesssim 10^{5} \mathrm{yr}$ ) down to $22 \pm 3 \%$ in pre-main sequence objects (age $\sim 10^{6} \mathrm{yr}$ ). Overall, we thus find evidence for an evolution of the multiplicity fraction during the embedded phase, with a decrease of multiple systems over a timescale of $10^{6} \mathrm{yr}$ at most, significant at the $2 \sigma$ level. The comparison between Class I sources with and without extended envelopes suggests that the actual timescale is of order $\sim 10^{5} \mathrm{yr}$.

We caution, however, that this result is limited by relatively small number statistics and strongly depends on the reliability of the evolutionary classification adopted for the YSOs in our sample. While the mass and/or optical depth of the envelope is generally recognized as being a robust tracer of the circumstellar evolution of embedded YSOs (e.g. Myers et al. 1998; André et al. 2000), it is more difficult to obtain reliable estimates of envelope properties in cluster-forming regions such as the Ophiuchus main cloud than in regions of "isolated" star formation such as Taurus. In Taurus, the relatively large spatial extent of prestellar cores and protostellar envelopes ( $\sim 18000 \mathrm{AU})$ makes it easy to distinguish between bona-fide protostars with envelopes and more evolved Class I sources (Motte \& André 2001). In Ophiuchus, however, the smaller prestellar fragmentation lengthscale leads to more compact protostellar envelopes which are more difficult to spatially resolve with single-dish millimeter telescopes. The current classification of Ophiuchus Class I sources in objects with or without extended envelopes (see Table 2, André \& Montmerle 1994; Motte et al. 1998) is thus more uncertain than in Taurus, pending the results of interferometric measurements in progress. If we consider the Taurus cloud alone, the decrease in the multiplicity fraction from self-embedded protostars to envelope-less objects becomes apparently stronger, although the sample size is then only barely statistically significant (see Table 4). In this framework, our results suggest a steep decrease of the fraction of multiple systems occurring during the protostellar embedded phase as the envelope is being dissipated on a timescale of $\sim 10^{5} \mathrm{yr}$.

\subsection{Confrontation with model predictions}

The above results indicate that the fraction of wide (110-1400 AU) protostellar multiple systems is initially high and seems to decrease on a timescale of $\sim 10^{5} \mathrm{yr}$ during the Class I phase in the Taurus and Ophiuchus clouds. How do these results compare with models of the formation and evolution of multiple systems? 
Assuming that the distribution of orbital periods of embedded multiple systems is at least roughly as wide as that of T Tauri and field binaries (Kroupa \& Burkert 2001), the multiplicity fraction we derive over a restricted range of separation suggests that, on average, each embedded YSO has at least one companion. This result is consistent with a class of models which rely on the assumption that all stars are initially in binary systems and investigate their subsequent dynamical evolution (e.g., Larson 1972; Kroupa 1995). Such models predict that the initially high fraction of multiple protostellar systems significantly decreases on a timescale of $1 \mathrm{Myr}$ or less in dense protostellar clusters, such as ONC, due to frequent disruptive encounters between systems (Kroupa et al. 2001), whereas the dynamical evolution is much milder in loose star-forming regions, such as Taurus, where gravitational encounters are rarer.

The high fraction of embedded multiple systems we report here for Taurus is consistent with the predictions of these models for low density regions. The small amount of dynamical evolution expected to occur in Taurus will take place very early on $\left(\leq 10^{5} \mathrm{yr}\right)$, when the initial protostellar aggregates still have a density of order $10^{3}$ stars $\mathrm{pc}^{-3}$ (Kroupa \& Bouvier 2003). Disruptive encounters between protostellar systems at this stage may result in the decreasing multiplicity fraction we observe in Taurus during the Class I phase on a timescale of $\sim 10^{5} \mathrm{yr}$ (Table 4 ). Past this early stage, the stellar aggregates quickly expand on a timescale of $\sim 10^{6} \mathrm{yr}$ to reach the currently observed densities of order 10-100 stars $\mathrm{pc}^{-3}$ and the Taurus population is not expected to dynamically evolve any more. Thus, the higher multiplicity rate of both embedded YSOs and $\mathrm{T}$ Tauri stars in Taurus with respect to field stars suggests that loose star-forming regions are not the dominant mode of star formation, i.e., that only a small proportion of all field stars form in such areas.

On the other hand, Ophiuchus is a young embedded cluster similar to the "dominant-mode cluster" of Kroupa's (1995a) models, from which most field stars are expected to arise (Clarke et al. 2000; Carpenter 2000; Adams \& Myers 2001). In Ophiuchus, as for Taurus, the initial decrease of multiplicity fraction we observe during the Class I embedded phase may be accounted for by disruptive encounters occurring in high density protostellar subclusters on a timescale of $\sim 10^{5} \mathrm{yr}$ (Bonnell et al. 2003). Unlike Taurus, however, the remaining excess of binaries observed among Class II-III T Tauri stars in Ophiuchus at an age of $\sim 10^{6} \mathrm{yr}$ has still to be reduced by a factor of two to be consistent with the frequency of field binaries. The current

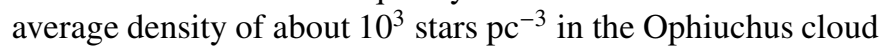
translates into a crossing time of order 1-2 Myr (e.g., Kroupa 1995a), which may indicate that the T Tauri population is not fully dynamically evolved yet in this cloud.

Together with observations of embedded multiple YSOs in an even denser cluster such as Orion, $N$-body simulations of the evolution of an Ophiuchus-like cluster would provide a crucial test of the assumption that core fragmentation produces a universal set of initial properties for protostellar multiple systems which subsequently evolve depending only upon environmental conditions. We note however that the similar frequency, properties and evolution we derive for embedded multiple systems in both Taurus and Ophiuchus seems to argue against large-scale environmental conditions playing a crucial role in shaping the properties of protostellar systems. Instead, the similar evolution of protostellar multiple systems in starforming regions whose average properties (e.g., mean stellar density) are so different may point to local conditions prevailing in e.g. small-scale embedded subclusters as being determinant (Bonnell et al. 2003; Sterzik et al. 2003).

The observed high frequency of multiple systems among embedded YSOs indicates that most prestellar cores undergo fragmentation into at least two objects during their collapse. It is often assumed that prestellar cores actually experience multiple fragmentation as they collapse thus leading to the formation of several protostellar seeds, i.e., small- $N$ protostellar aggregates with $N=3-10$, typically, on a scale of a few $100 \mathrm{AU}$ (e.g. Burkert et al. 1997; Sterzik \& Durisen 1998; Delgado-Donate et al. 2003; Goodwin et al. 2004a). Global simulations of cloud collapse support this scenario and predict the formation of even higher density subclusters consisting of 10-50 protostellar seeds (Klessen et al. 1998; Bate et al. 2003; Bonnell et al. 2003). Small- $N$ protostellar aggregates as well as high density subclusters undergo rapid dynamical evolution on a timescale of $10^{4-5}$ yr (Sterzik \& Durisen 1998; Delgado-Donate et al. 2003). The dynamical decay of initially unstable groups is thus expected to eject most of the lowest mass protostellar seeds, with a typical ejection velocity of $\lesssim 1 \mathrm{~km} \mathrm{~s}^{-1}$, while those remaining in the cloud core form stable binary and higher order multiple systems.

At an age of about $10^{5} \mathrm{yr}$, we find no evidence that embedded YSOs in Taurus and Ophiuchus belong to small- $N$ clusters or high density subclusters on a scale of a few 100 AU. No triple embedded YSO is found in Taurus, and only two in Ophiuchus, compared to a total of 13 binary YSOs detected over the separation range 110-1400 AU. It may be argued that dynamical evolution of small- $N$ clusters has already taken place by that time, ejecting most of the initial protostellar seeds at larger distances ( $\gg 1000 \mathrm{AU}$ ) and leaving only the resulting stable systems. However, Allen et al. (2002) investigated the spatial distribution of prestellar cores and YSOs in Ophiuchus and concluded that the YSOs had neither concentrated nor dispersed significantly since their formation in these cloud cores. Numerical simulations of the dynamical decay of small- $N$ clusters including accretion onto the protostellar seeds and circumstellar disks (e.g. Delgado-Donate et al. 2003; Goodwin et al. 2004a) predict the formation of an equal number of tight binaries and higher order (triple and quadruple) systems. This prediction also seems to contrast with our results, although it may simply indicate that a fraction of our sample primaries actually are tight binaries with a separation less than $100 \mathrm{AU}$ and have therefore gone unresolved in this study. Pending a higher resolution study of these embedded sources (Duchêne et al., in prep.), a comparison of the hierarchy of multiplicity as derived from observations with model predictions remains somewhat inconclusive.

For the time being, on the sole basis of the high frequency of wide binaries among embedded Class I and flat spectrum sources, we cannot firmly distinguish between the formation of unstable small- $N$ aggregates and a core fragmentation process that only creates two or three seeds, i.e. stable multiple systems 
without further internal dynamical evolution. In any event, the similar multiplicity rate we derive for embedded YSOs in both Taurus and Ophiuchus in spite of initially different prestellar core sizes and widely different average stellar density between the two star-forming regions indicates that, regardless of the formation channel, the outcome is fairly insensitive to both initial and environmental conditions in this range. Whether this is accounted for by current models remains to be seen (e.g., Delgado-Donate et al. 2004; Goodwin et al. 2004b; Sterzik et al. 2003).

\section{Conclusions}

We have conducted a survey of visual companions to $63 \mathrm{em}$ bedded YSOs in the Taurus-Auriga and Ophiuchus starforming regions. The main results are as follows:

- The companion star fraction of Class I and flat spectrum embedded sources over a separation range 110-1400 AU is $23 \pm 9 \%$ in Taurus, $29 \pm 7 \%$ in Ophiuchus and $27 \pm 6 \%$ for the combined sample. This fraction is twice as large as the frequency of companions to $\mathrm{G}$ dwarfs in the solar neighborhood, in the same separation range. Assuming that the distribution of orbital periods of protostellar multiple systems is about as wide as that of mature systems, this high multiplicity fraction suggests that each embedded YSO has, on average, at least one companion. The fragmentation of prestellar cores as they collapse thus appears to be a frequent occurrence indeed.

- In spite of the known differences in the properties of prestellar cores in Taurus and Ophiuchus and the widely different stellar density in the two star-forming regions, we find identical properties for their embedded multiple systems (frequency, separations, luminosity ratios). This suggests that the outcome of the fragmentation of prestellar cores is fairly insensitive to both initial and environmental conditions, at least for the range of physical conditions encountered in these two clouds.

- Combining the Taurus and Ophiuchus clouds, the fraction of multiple systems appears to decrease on a timescale of order $\sim 10^{5} \mathrm{yr}$ during the embedded phase, with the youngest embedded sources, Class 0 and Class I YSOs still surrounded by extended millimetric envelopes, exhibiting nearly twice as many multiple systems as more evolved Class I YSOs devoid of $\mathrm{mm}$ envelopes and Class II-III $\mathrm{T}$ Tauri stars. This suggests that rapid disruption of wide protostellar multiple systems occurs during the embedded phase, resulting either from the internal decay of initially unstable systems or from disruptive encounters between stable systems in high density protostellar aggregates.

- Over a scale of a few 100 AU, embedded YSOs at an age $\sim 10^{5} \mathrm{yr}$ in Taurus and Ophiuchus clouds are found to be in relative isolation and not assembled in subclusters. This seems to contrast with the assumption that protostellar collapse leads to small- $N$ clusters $(N=3-10)$ as well as with global simulations of cloud collapse which predict the formation of even higher density subclusters on these scales. Evidence for such clustering properties may have to be searched for on either smaller scales (tight binaries) or larger ones (ejected protostellar seeds) if dynamical evolution has already occurred.

Taurus and Ophiuchus clouds probe different modes of star formation, the former being a low density association harboring only low mass objects, while the latter is a dense cluster including at least one massive B star as well as a large population of low mass $\mathrm{T}$ Tauri stars. The results reported here unexpectedly suggest that the properties and early evolution of embedded multiple systems in these two clouds are quite similar and therefore do not seem to depend much on initial and/or global environmental conditions. This may indicate that the properties of multiple systems are ultimately driven by dynamical processes acting on small scales and are thus shaped by local (rather than global) conditions which might not be very different between clusters and associations during the early stages of protostellar evolution.

Acknowledgements. We are grateful to Bo Reipurth and to an anonymous referee for enriching comments that helped us clarify this manuscript. We extend our thanks to the CFHT and ESO/NTT staffs who supported us very efficiently during our observing runs. This study has made use of the SIMBAD database, operated at CDS, Strasbourg, France, and of the Two Micron All Sky Survey, which is a joint project of the University of Massachussetts and the Infrared Processing and Analysis Center, funded by the National Aeronautics and Space Administration and the National Science Foundation. Partial funding from the European Research Training Network on "The Formation and Evolution of Young Stellar Clusters" (HPRN-CT-2000-00155) is ackowledged.

\section{Appendix A: T Tauri stars in Ophiuchus}

In the course of our survey, we observed many objects that are members of the Ophiuchus star-forming regions but are in the more evolved classical and weak-line T Tauri (Class II and III respectively) stages. For completeness, we list here all the objects we observed (Table A.1) as well as the candidate companions detected within $10^{\prime \prime}$ of them (Table A.2). Among the companions listed in Table A.2, only three were previously known, namely the ISO 60-61 pair (listed in Bontemps et al. 2001) and the companions to ISO 95 and ISO 204, listed in Haisch et al. (2002).

It can be noted that we detected a much larger fraction of candidate companions among this sample of T Tauri stars than among the protostars that form the primary sample of this study $(51 \pm 6 \%$ vs. $30 \pm 6 \%$ ). This is most likely a consequence of the fact that the protostars are located deeper in the molecular cloud and therefore they are surrounded by comparatively darker areas in our images. On the other hand, most T Tauri stars are located in areas that probably contain many background stars that are not completely extincted, which leads to a larger number of projected visual pairs. This is confirmed by the generally large flux ratios between the T Tauri stars and their apparent companions: almost $75 \%$ of all pairs listed in Table A. 2 have $\Delta K \geq 5$ mag while only three such pairs are found in Table 3 . 
Table A.1. List of all T Tauri stars (Class II and III objects) in Ophiuchus that were detected in the vicinity of our target protostars. Objects whose name starts with "B" are from Barsony et al. (1997). The classification is determined from the infrared spectral indices following Bontemps et al. (2001).

\begin{tabular}{|c|c|c|c|c|c|c|c|c|}
\hline ISO\# & Object & Class & ISO\# & Object & Class & ISO\# & Object & Class \\
\hline 9 & SKS 1-4 & II & 76 & GY 146 & II & 136 & GY 258 & III \\
\hline 13 & B $162607-242725$ & II & 78 & VSSG 5/GY 153 & II & 140 & GY 262 & II \\
\hline 14 & В $162607-242742$ & III & 79 & GY 154 & II & 142 & VSSG 25/GY 267 & II \\
\hline 19 & GSS 29 & II & 81 & VSSG 7/GY 157 & III & 144 & IRS 45/GY 273 & II \\
\hline 22 & B $162618-241712$ & III & 82 & GY 163 & III & 150 & ISO 1627309-242734 & II \\
\hline 23 & SKS $1-10$ & II & 84 & WL 21/GY 164 & II & 154 & GY 291 & II \\
\hline 24 & VSSG 1 & II & 86 & IRS 26/GY 171 & II & 155 & GY 292 & II \\
\hline 25 & CRBR 17 & III & 87 & В $162658-241836$ & II & 156 & GY 295 & III \\
\hline 28 & В $162621-241544$ & III & 89 & WL 14/GY 172 & II & 164 & GY 310 & II \\
\hline 30 & GY 5 & II & 90 & WL 22/GY 174 & II & 166 & GY 314 & II \\
\hline 35 & GY 215 & II & 91 & VSSG 8/GY 181 & III & 171 & GY 323 & II \\
\hline 37 & LFAM 3/GY 21 & II & 92 & WL 16/GY 182 & II & 172 & GY 326 & II \\
\hline 39 & S $2 / G Y 23$ & II & 93 & GY 188 & II & 173 & IRS 53/GY 334 & III \\
\hline 40 & $\mathrm{El} 24$ & II & 94 & В $162703-242007$ & II & 176 & GY 350 & II \\
\hline 41 & GY 29 & II & 95 & WL 1/GY 192 & II & 177 & GY 352 & II \\
\hline 42 & VSSG 29/GY 37 & III & 98 & GY 195 & II & 179 & GY 370 & III \\
\hline 48 & S $1 / G Y 70$ & III & 100 & В $162705-244013$ & III & 183 & GY 377 & III \\
\hline 51 & В $162636-241554$ & II & 101 & IRS 30/GY 203 & III & 189 & GY 412 & III \\
\hline 52 & VSSG 4/GY 81 & II & 104 & GY 207 & III & 190 & GY 450 & II \\
\hline 57 & В $162641-241801$ & III & 107 & GY 213 & II & 191 & GY 463 & III \\
\hline 58 & WL 8/GY 96 & III & 114 & WL 19/GY 227 & II/III & 192 & GY 472 & III \\
\hline 60 & GY 101 & III & 120 & IRS 34/GY 239 & II & 201 & ISO $1631487-245432$ & II \\
\hline 64 & VSSG 11 & III & 122 & IRS 36/GY 241 & II & 204 & L1689-IRS 5 & II \\
\hline 71 & GY 130 & III & 125 & WL 5/GY 246 & III & 205 & ISO $1631531-245504$ & II \\
\hline 72 & WL 18/GY 129 & II & 126 & GY 248 & III & 208 & ISO $1631591-245442$ & II \\
\hline 74 & IRS 20/GY 143 & III & 130 & SR 12/GY 250 & III & & & \\
\hline
\end{tabular}

Table A.2. List of all companions to T Tauri stars in Ophiuchus that were detected in the course of our survey.

\begin{tabular}{|c|c|c|c|c|c|c|c|c|c|c|c|}
\hline ISO\# & $\begin{array}{c}\rho \\
\left({ }^{\prime \prime}\right)\end{array}$ & $\begin{array}{l}\mathrm{PA} \\
\left({ }^{\circ}\right)\end{array}$ & $\begin{array}{c}\Delta H \\
(\mathrm{mag})\end{array}$ & $\begin{array}{c}\Delta K \\
(\mathrm{mag})\end{array}$ & Note & ISO\# & $\begin{array}{c}\rho \\
\left({ }^{\prime \prime}\right)\end{array}$ & $\begin{array}{l}\text { PA } \\
\left({ }^{\circ}\right)\end{array}$ & $\begin{array}{c}\Delta H \\
(\mathrm{mag})\end{array}$ & $\begin{array}{c}\Delta K \\
(\mathrm{mag})\end{array}$ & Note \\
\hline 9 & 7.18 & 240.0 & & $6.74 \pm 0.10$ & & 95 & 0.87 & 322.8 & $0.10 \pm 0.10$ & $0.01 \pm 0.03$ & \\
\hline 23 & 9.92 & 154.4 & & $5.45 \pm 0.05$ & & 126 & 5.60 & 214.7 & & $>6.0$ & $\mathrm{~b}$ \\
\hline- & 7.54 & 118.8 & & $7.1 \pm 0.2$ & & 130 & 8.76 & 162.9 & & $6.37 \pm 0.05$ & \\
\hline 24 & 6.68 & 102.4 & & $8.51 \pm 0.10$ & & 142 & 8.72 & 312.5 & $>5.2$ & $6.25 \pm 0.10$ & \\
\hline- & 7.64 & 131.1 & & $10.1 \pm 0.2$ & & 154 & 7.86 & 196.5 & $2.7 \pm 0.2$ & $2.00 \pm 0.03$ & \\
\hline 30 & 8.19 & 19.9 & & $7.82 \pm 0.10$ & & 156 & 6.31 & 84.1 & & $7.13 \pm 0.10$ & \\
\hline 35 & 7.37 & 282.4 & & $7.7 \pm 0.2$ & & 166 & 3.30 & 102.2 & & $5.9 \pm 0.2$ & \\
\hline 41 & 7.76 & 273.1 & & $3.54 \pm 0.10$ & & 172 & 9.81 & 254.0 & & $6.20 \pm 0.05$ & \\
\hline 42 & 7.90 & 341.0 & & $6.80 \pm 0.15$ & & - & 6.44 & 100.4 & & $7.48 \pm 0.10$ & \\
\hline 51 & 6.06 & 56.6 & & $7.8 \pm 0.2$ & & - & 8.07 & 80.4 & & $5.18 \pm 0.05$ & \\
\hline- & 6.28 & 104.0 & & $6.94 \pm 0.10$ & & 176 & 2.23 & 161.4 & $6.6 \pm 0.2$ & $5.15 \pm 0.05$ & \\
\hline 52 & 7.39 & 90.7 & $>5.9$ & $7.61 \pm 0.15$ & & - & 3.34 & 55.8 & $7.1 \pm 0.2$ & $5.89 \pm 0.05$ & \\
\hline 57 & 1.51 & 241.6 & & $1.47 \pm 0.10$ & & 183 & 6.21 & 256.2 & & $6.68 \pm 0.05$ & \\
\hline 60 & 5.99 & 348.4 & $-0.85 \pm 0.03$ & $0.01 \pm 0.02$ & $\mathrm{a}$ & - & 8.71 & 261.2 & & $7.6 \pm 0.2$ & \\
\hline 64 & 4.9 & 134 & & $>7.2$ & $\mathrm{~b}$ & 189 & 6.78 & 115.9 & & $7.5 \pm 0.2$ & \\
\hline 72 & 3.57 & 291.1 & & $1.78 \pm 0.05$ & & - & 7.20 & 113.0 & & $7.5 \pm 0.2$ & \\
\hline 74 & 1.04 & 206.6 & & $2.7 \pm 0.2$ & & 190 & 8.01 & 84.4 & & $6.17 \pm 0.15$ & \\
\hline 81 & 7.97 & 186.8 & & $6.49 \pm 0.10$ & & 204 & 2.95 & 241.4 & & $0.30 \pm 0.10$ & $\mathrm{c}$ \\
\hline 82 & 9.25 & 204.5 & $>2.7$ & $4.50 \pm 0.10$ & & 05 & 6.39 & 318.8 & & $6.2 \pm 0.1$ & \\
\hline 86 & 7.28 & 78.3 & & $7.8 \pm 0.2$ & & & & & & & \\
\hline
\end{tabular}

Notes - a: The companion to this source is ISO 61 ( $\equiv$ GY 103), a Class III object.

b: The primary is saturated at $K$ band; no observation is available in $H$ band.

c: The primary is slightly saturated. 


\section{References}

Adams, F. C., \& Myers, P. C. 2001, ApJ, 553, 744

Allen, L. E., Myers, P. C., Di Francesco, J., et al. 2002, ApJ, 566, 993

André, P., \& Montmerle, T. 1994, ApJ, 420, 837

André, P., Ward-Thomson, D., \& Barsony, M. 2000, in Protostars and Planets IV, ed. V. Mannings, A. P. Boss, \& S. S. Russell (Tucson: University of Arizona Press), 59

Barsony, M., Kenyon, S. J., Lada, E. A., \& Teuben, P. J. 1997, ApJS, 112,109

Barsony, M., Koresko, C., \& Matthews, K. 2003, ApJ, 591, 1064

Bate, M. 2000, MNRAS, 314, 33

Bate, M. R., Bonnell, I. A., \& Bromm, V. 2003, MNRAS, 339, 577

Bertout, C., Robichon, N., \& Arenou, F. 1999, 352, 574

Bonnell, I., Arcoragi, J.-P., Martel, H., \& Bastien, P. 1992, ApJ, 400, 579

Bonnell, I. A., Bate, M. R., \& Vine, S. G. 2003, MNRAS, 343, 413

Bontemps, S., André, P., Kaas, A. A., et al. 2001, A\&A, 372, 173

Boss, A. P. 2002, ApJ, 576, 462

Burkert, A., Bate, M. R., \& Bodenheimer, P. 1997, MNRAS, 289, 497

Carpenter, J. M. 2000, AJ, 120, 3139

Clarke, C. J., Bonnell, I. A., \& Hillenbrand, L. A. 2000, in Protostars and Planets IV, ed. V. Mannings, A. P. Boss, \& S. S. Russell (Tucson: University of Arizona Press), 151

Delgado-Donate, E. J., Clarke, C. J., \& Bate, M. R. 2003, MNRAS, 342,926

Delgado-Donate, E. J., Clarke, C. J., \& Bate, M. R. 2004, MNRAS, 347,759

Duchêne, G. 1999, A\&A, 341, 547

Duchêne, G., Simon, T., Eislöffel, J., \& Bouvier, J. 2001, A\&A, 379, 147

Duquennoy, A., \& Mayor, M. 1991, A\&A, 248, 485

Durisen, R. H., \& Sterzik, M. F. 1994, A\&A, 286, 84

Gomez, M., Hartmann, L., Kenyon, S. J., \& Hewett, R. 1994, AJ, 105, 1927

Goodwin, S. P., Whitworth, A. P., \& Ward-Thompson, D. 2004a, A\&A, 414, 633

Goodwin, S. P., Whitworth, A. P., \& Ward-Thompson, D. 2004b, A\&A, 419, 543

Greene, T. P., Wilking, B. A., André, P., Young, E. T., \& Lada, C. J. 1994, ApJ, 434, 614
Haisch, K. E., Barsony, M., Greene, T. P., \& Ressler, M. E. 2002, AJ, 124, 2841

Haisch, K. E., Greene, T. P., Barsony, M., \& Stahler, S. W. 2004, AJ, 127,1747

Kenyon, S. J., \& Hartmann, L. 1995, ApJS, 101, 117

Klessen, R. S., Burkert, A., \& Bate, M. R. 1998, ApJ, 501, L205

Kroupa, P. 1995a, MNRAS, 277, 1491

Kroupa, P. 1995b, MNRAS, 277, 1522

Kroupa, P., Petr, M. G., \& McCaughrean, M. J. 1999, New A., 4, 495

Kroupa, P., \& Burkert, A. 2001, ApJ, 555, 945

Kroupa, P., Aarseth, S., \& Hurley, J. 2001, MNRAS, 321, 699

Kroupa, P., \& Bouvier, J. 2003, MNRAS, 346, 343

Larson, R. B. 1972, MNRAS, 156, 437

Leinert, C., \& Haas, M. 1989, ApJ, 342, L39

Leinert, C., Zinnecker, H., Weitzel, N., et al. 1993, A\&A, 278, 129

Looney, L. W., Mundy, L. G., \& Welch, W. J. 2000, ApJ, 529, 477

Mathieu, R. D. 1994, ARA\&A, 32, 465

Meyer, M. R. 1996, Ph.D. Thesis, Univeristy of Massachusetts

Motte, F. 1998, Ph.D. Thesis, Univeristy Paris XI

Motte, F., André, P., \& Neri, R. 1998, A\&A, 336, 150

Motte, F., \& André, P. 2001, A\&A, 365, 440

Myers, P. C., Adams, F. C., Chen, H., \& Schaff, E. 1998, ApJ, 492, 703

Padgett, D. L., Brandner, W., Stapelfeldt, K. R., et al. 1999, AJ, 117, 1490

Park, S., \& Kenyon, S. J. 2002, AJ, 123, 3370

Reipurth, B., Yu, K. C., Rodríguez, L. F., Heathcote, S., \& Bally, J. 1999, A\&A, 352, L83

Reipurth, B. 2000, AJ, 120, 3177

Reipurth, B., Rodríguez, L. F., Anglada, G., \& Bally, J. 2002, AJ, 124, 1045

Reipurth, B., Rodríguez, L. F., Anglada, G., \& Bally, J. 2004, AJ, 127, 1746

Ressler, M. E., \& Barsony, M. 2001, AJ, 121, 1098

Rieke, G. H., \& Lebofsky, M. J. 1985, ApJ, 288, 618

Starr, B. M., Doyon, R., Beuzit, J.-L., et al. 2000, SPIE, 4008, 999

Sterzik, M. F., \& Durisen, R. H. 1998, A\&A, 339, 95

Sterzik, M. F., Durisen, R. H., \& Zinnecker, H. 2003, A\&A, 411, 91 